\title{
Cdk5 Regulation of the GRAB-Mediated Rab8-Rab11 Cascade in Axon Outgrowth
}

\author{
Kotaro Furusawa, ${ }^{1}$ Akiko Asada, ${ }^{1}$ Pamela Urrutia, ${ }^{2,3}$ Christian Gonzalez-Billault, ${ }^{2,3,4}$ Mitsunori Fukuda, ${ }^{5}$ \\ and ${ }^{\circledR S h i n}$-ichi Hisanaga ${ }^{1}$ \\ ${ }^{1}$ Department of Biological Sciences, Graduate School of Science, Tokyo Metropolitan University, Minami-Osawa, Hachioji, Tokyo 192-0397, Japan, \\ ${ }^{2}$ Department of Biology, Faculty of Sciences, Universidad de Chile, 7800024 Nunoa, Chile, ${ }^{3}$ FONDAP Geroscience Center for Brain Health and Metabolism, \\ 7500922 Santiago, Chile, ${ }^{4}$ Buck Institute for Research on Aging, Novato, California 94945, and ${ }^{5}$ Department of Developmental Biology and Neurosciences, \\ Graduate School of Life Sciences, Tohoku University, Aoba-ku, Sendai, Miyagi 980-8578, Japan
}

Neurons communicate with each other through their axons and dendrites. However, a full characterization of the molecular mechanisms involved in axon and dendrite formation is still incomplete. Neurite outgrowth requires the supply of membrane components for surface expansion. Two membrane sources for axon outgrowth are suggested: Golgi secretary vesicles and endocytic recycling endosomes. In non-neuronal cells, trafficking of secretary vesicles from Golgi is regulated by Rab8, a member of Rab small GTPases, and that of recycling endosomes is by Rab11, another member of Rabs. However, whether these vesicles are coordinately or independently transported in growing axons is unknown. Herein, we find that GRAB, a guanine nucleotide exchange factor for Rab8, is a novel regulator of axon outgrowth. Knockdown of GRAB suppressed axon outgrowth of cultured mouse brain cortical neurons. GRAB mediates the interaction between Rab11A and Rab8A, and this activity is regulated by phosphorylation at Ser169 and Ser180 by Cdk5-p35. The nonphosphorylatable GRAB mutant S169/180A promoted axonal outgrowth to a greater extent than did the phosphomimetic GRAB mutant S169/180D. Phosphorylation of GRAB suppressed its guanine nucleotide exchange factor activity and its ability to recruit Rab8A- to Rab11A-positive endosomes. In vivo function of GRAB and its $\mathrm{Cdk} 5$-phophorylation were shown in migration and process formation of developing neurons in embryonic mouse brains. These results indicate that GRAB regulates axonal outgrowth via activation and recruitment of Rab8A- to Rab11A-positive endosomes in a Cdk5-dependent manner.

Key words: axon outgrowth; Cdk5; GRAB; phosphorylation; Rab; vesicle transport

Significance Statement

While axon outgrowth requires membrane supply for surface expansion, the molecular mechanisms regulating the membrane transport in growing axons remain unclear. Here, we demonstrate that GRAB, a guanine nucleotide exchange factor for Rab8, is a novel regulator of axon outgrowth. GRAB promotes the axonal membrane transport by mediating the interaction between Rab11 and Rab8 in neurons. The activity of GRAB is regulated by phosphorylation with Cdk5. We describe an in vivo role for GRAB and its Cdk5 phosphorylation during neuronal migration and process formation in embryonic brains. Thus, the membrane supply for axonal outgrowth is regulated by Cdk5 through the Rab11-GRAB-Rab8 cascade.

\section{Introduction}

Neurons communicate with each other through their axons and dendrites, which are structurally and functionally distinct. The proper elongation timing, speed, and direction of these processes

\footnotetext{
Received July 11, 2016; revised Nov. 21, 2016; accepted Nov. 28, 2016.

Author contributions: K.F. and S.-i.H. designed research; K.F. and P.U. performed research; A.A. and M.F. contributed unpublished reagents/analytic tools; K.F., A.A., C.G.-B., M.F., and S.-i.H. analyzed data; K.F., C.G.-B., and S.-i.H. wrote the paper.

This work was supported in part by MEXT in Japan Grants-in-Aid for Scientific Research on Priority Area 25290024 and 26117004 to S.H. and 15 H04367 and 15H01198 to M.F., Grants Fondecyt 1140325 and FONDAP 15150012 to C.G.-B., and postdoctoral Fondecyt Grant 3160630 to P.U.

The authors declare no competing financial interests.
}

need to be tightly controlled. Dysregulation of process formation causes detrimental brain diseases because of improper neuronal connections (Bellon, 2007; Bakos et al., 2015). Because axon and dendrite formation can be recapitulated in cultured neurons (Dotti et al., 1988), many studies have attempted to elucidate the underlying molecular mechanisms of process formation. Although the roles of cytoskeletal proteins have been well docu-

Correspondence should be addressed to either Dr. Kotaro Furusawa or Dr. Shin-ichi Hisanaga, Department of Biological Sciences, Graduate School of Science, Tokyo Metropolitan University, Minami-Osawa, Hachioji, Tokyo 192-0397, Japan. E-mail: furusawa-kotaro@ed.tmu.ac.jp or hisanaga-shinichi@tmu.ac.jp. DOI:10.1523/JNEUROSCI.2197-16.2016

Copyright $\odot 2017$ the authors $\quad 0270-6474 / 17 / 370790-17 \$ 15.00 / 0$ 
mented during process formation (Conde and Cáceres, 2009; Lewis et al., 2013), only recently the regulation of membrane transport at the molecular level has become a research target (Sann et al., 2009). Although some evidence is available suggesting that membrane transport is important for neuronal differentiation (Villarroel-Campos et al., 2014, 2016a), the underlying delivery mechanisms responsible for specific axonal or dendritic membrane formation have not been elucidated.

Membrane trafficking is a dynamic process involving vesicle formation in donor compartments for transport and their delivery, tethering, and docking to their acceptor membranes (Takai et al., 2001; Zerial and McBride, 2001; Stenmark, 2009). The small GTPases of the Rab family regulate these processes. More than 60 Rabs are known, each of which is located and functions in a distinct membrane compartment (Klöpper et al., 2012). Their activity cycles between an activated form bound to GTP and an inactive form bound to GDP (Stenmark, 2009). Interconversion of the active and inactive states is mediated by guanine nucleotide exchange factors (GEFs), which exchange GDP for GTP and GTPase-activating proteins (GAPs), which stimulate GTP hydrolysis. Therefore, the regulation mechanism of each GEF or GAP for each Rab must be individually described, but only a few cases have been reported (Xu et al., 2015).

Membranes are required for surface expansion during axon growth and are supplied by exocytic (secretory) and endocytic pathways (Horton and Ehlers, 2003; Pfenninger, 2009). In the secretory pathway, Rab8 regulates the transport of exocytic vesicles from the trans-Golgi network to peripheral membranes (Stenmark, 2009). A deficiency in Rab8 activity results in decreased neurite outgrowth in embryonic hippocampal neurons (Huber et al., 1995). In the endocytic pathway, which requires several Rabs (e.g., Rab5 and Rab11), plasma membranes are endocytosed and then processed as recycling vesicles, which are delivered back to the cell surface (Takai et al., 2001; Zerial and McBride, 2001). Rab11 regulates endosome transport to axon tips (Ascano et al., 2009; Eva et al., 2010; Takano et al., 2012). However, whether these endocytic and exocytic Rabs act independently or cooperatively during axon outgrowth is not known. We had an interest in GRAB, a GEF for Rab8 (Yoshimura et al., 2010; Guo et al., 2013), which was proposed to have a role in synaptic vesicle recycling in the presynaptic region (Luo et al., 2001), because GRAB also interacts with Rab11 (Horgan et al., 2013). However, the role of GRAB in axon outgrowth has not been addressed at all.

Cdk5 is a proline-directed Ser/Thr protein kinase that can be activated by binding the regulatory subunit of neuronal, membrane-bound p35 or p39 (Hisanaga and Endo, 2010). Cdk5 is active mainly in postmitotic neurons and is involved in a variety of neuronal functions (e.g., neuronal migration and axonal outgrowth) (Smith and Tsai, 2002; Kawauchi, 2014). Recently, we have shown that Cdk5-p35 regulates trafficking of Rab11Apositive recycling endosomes via lemur kinase 1A (LMTK1A) during axon outgrowth (Takano et al., 2012, 2014). However, other molecular mechanisms regulated by Cdk5-p35 and involving membrane delivery to supoport axonal outgrowth are currently unknown.

Herein, we first validate that GRAB is a GEF of Rab8 and then show that it mediates the interaction between Rab11 and Rab8 in neurons. We find that Cdk5-p35 regulates GRAB activity by phosphorylation, thereby regulating axonal outgrowth via a Rab11-GRAB-Rab8 cascade.

\section{Materials and Methods}

Antibodies and plasmids. Anti-p35 C19 (Santa Cruz Biotechnology, catalog \#sc-820, RRID:AB_632137), anti-Cdk5 C-8 (Santa Cruz Biotechnology, catalog \#sc-173, RRID:AB_631224) or DC17 (Millipore, catalog \#05-364, RRID:AB_2229170), and control IgG (Santa Cruz Biotechnology, catalog \#sc-2025, RRID:AB_737182) were obtained from Santa Cruz Biotechnology. Anti-Rab8 (catalog \#6975S, RRID:AB_10827742) was purchased from Cell Signaling Technology. Anti-Rab11 (catalog \#610656, RRID:AB_397983) was from BD Biosciences. Anti-myc 4A6 (catalog \#05-724, RRID:AB_309938) was purchased from Millipore. Mouse monoclonal anti-GFP antibody (catalog \#11814460001, RRID: AB_390913) was obtained from Roche Diagnostics. Rabbit polyclonal anti-GFP antibody (catalog \#598, RRID:AB_591819) was from MBL International. Anti-actin (catalog \#A2066, RRID:AB_476693) was purchased from Sigma-Aldrich. Biotinylated anti-rabbit IgG (catalog \#BA-1000, RRID:AB_2313606) was from Vector Laboratories. AntiGRAB rabbit polyclonal antibody was obtained using purified GSTGRAB as the antigen and affinity purifying it from the resulting antiserum using antigen-immobilized beads (Fukuda and Mikoshiba, 1999). Secondary antibodies conjugated with AlexaFluor-546 (Thermo Fisher Scientific, catalog \#A-11010, RRID:AB_2534077) or AlexaFluor647 (Thermo Fisher Scientific, catalog \#A-21244, RRID:AB_2535812) were purchased from Invitrogen.

The plasmid encoding HA-Cdk5, kinase-negative HA-Cdk5, or myc-p35, was described previously (Asada et al., 2008; Kaminosono et al., 2008). The plasmids, pEGFP-Rab8A, pEGFP-Rab11A, their constitutively active (ca) and constitutively inactive (ci)-containing mutants, and pmStr-Rab11A were described previously (Fukuda, 2003; Sano et al., 2008; Mori et al., 2012). pEGFP-GRAB, pmStr-GRAB, and pEF-GRAB were prepared as described previously (Mori et al., 2013). pGEX-MICAL-L2-CC was isolated as described previously (Sano et al., 2008). The nonphosphorylatable Ala (A) and the phosphorylation-mimic Asp (D) mutants of GRAB were constructed using pmStr-GRAB or pEGFP-GRAB as the template as follows: $5^{\prime}$-ACAC CAGCCGCTCCCAACCGT-3' and 5'-ACGGTTGGGAGCGGCTGGTGT$3^{\prime}$ as forward and reverse primers for the S169A mutant; $5^{\prime}$-ACACCA GCCGATCCCAACCGT- ${ }^{\prime}$ and 5' $^{\prime}$-ACGGTTGGGATCGGCTGGT GT $-3^{\prime}$ as forward and reverse primers for the S169D mutant; $5^{\prime}$-CAG CTGCTGGCCCCCACCAAA- $3^{\prime}$ and 5' $^{\prime}$-TTTGGTGGGGGCCAGC AGCTG- $3^{\prime}$ as forward and reverse primers for the S180A mutant; $5^{\prime}$ CAGCTGCTGGACCCCACCAAA- $3^{\prime}$ and $5^{\prime}$-TTTGGTGGGGTCCAG CAGCTG- $3^{\prime}$ as forward and reverse primers for the S180D mutant; $5^{\prime}$ CATATCCCTGCCCCTGACAAA- $3^{\prime}$ and $5^{\prime}$-TTTGTCAGGGGCAG GGATATG- $3^{\prime}$ as forward and reverse primers for the T213A mutant; $5^{\prime}$ ACACCAGCCGATCCCAACCGT-3' and 5'-ACGGTTGGGATCGGCTG GTGT- $3^{\prime}$ as forward and reverse primers for the S169A mutant; and $5^{\prime}-$ CAGCTGCTGGACCCCACCAAA- ${ }^{\prime}$ and $5^{\prime}$-TTTGGTGGGGTCCAGC AGCTG $-3^{\prime}$ as forward and reverse primers for the S180A mutant. The GRAB knockdown shRNA sequences were $5^{\prime}$-GTATGCAACTTCTTCACCTAT-3' for shGRAB \#1; 5' -CTTCTTCACCTATATTCGCTA-3' for shGRAB \#2; 5' GAGCTGAAGCTAAAGGATGAA-3' for shGRAB \#3; 5' -GCCCACTGTT GAGTGTAACAA-3' for shGRAB \#4; and 5'-ATCCTTATAGGCCA TCTCTTA- $3^{\prime}$ for the scramble sequence (SC). Each knockdown sequence was individually inserted into a pSUPER.puro vector (Oligoengine).

Antibodies against GRAB phospho-Ser169 and phospho-Ser180. The phospho-Ser169 peptide CTSTPA(pS)PNRE, phospho-Ser180 peptide HPQLL(pS)PTKA, non-phospho-Ser169 peptide CTSTPASPNRE, non-phospho-Ser180 peptide HPQLLSPTKA, the keyhole limpet hemocyanin-conjugated phospho-Ser169 peptide, and the keyhole limpet hemocyanin-conjugated phospho-Ser180 peptide were obtained from GenScript. Female rabbits (New Zealand White, 14-15 weeks old; Sankyo Labo) were inoculated with one of the keyhole limpet hemocyanin-conjugated peptides using TiterMax Gold (Funakoshi) as the adjuvant. Anti-phospho-Ser180 was purified from the rabbit antiserum using the phospho-Ser 180 peptide-affinity column after removing non-phospho-antibody over the corresponding nonphospho-peptideaffinity column. The phospho-Ser169 antiserum was used for immunoblotting without purification because the anti-phospho-Ser169 lost activity upon purification. 

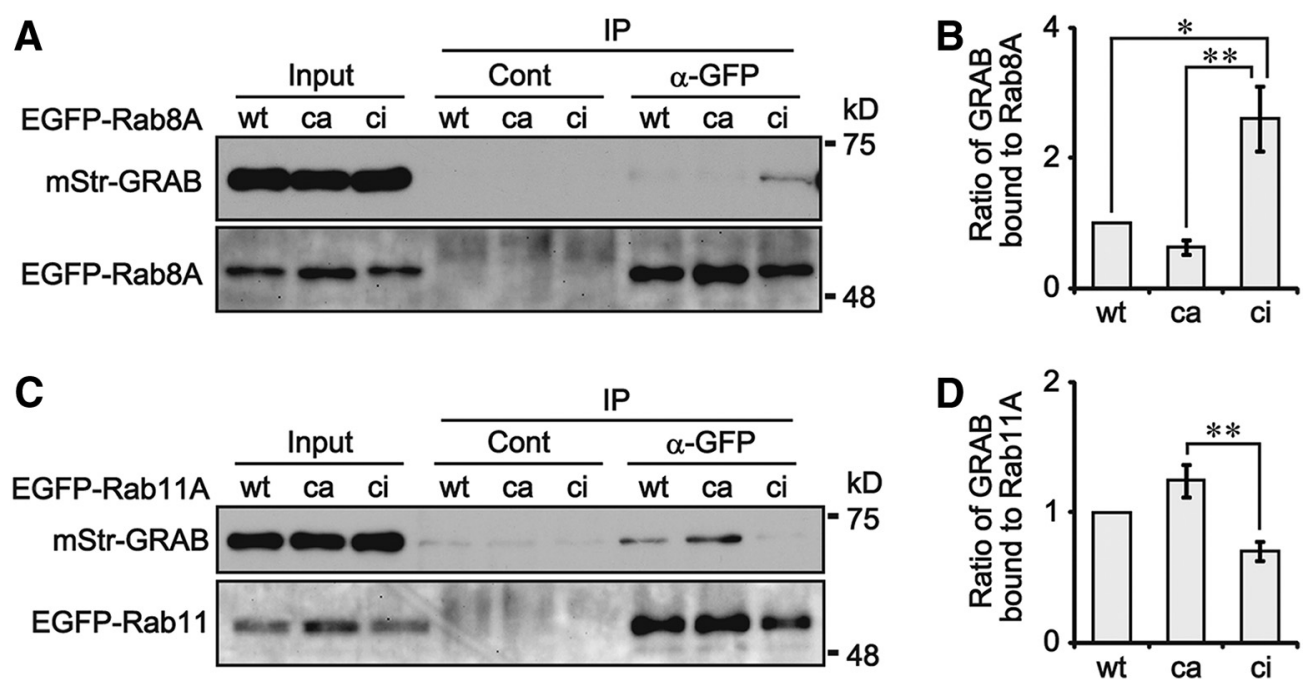

Figure 1. Interaction of GRAB with Rab8A or Rab11A. A, Preferential association of ci Rab8A with GRAB. The extracts (input) of COS-7 cells expressing mStr-GRAB and wt Rab8A, ca Rab8A, or ci Rab8A (EGFP-tagged) were immunoprecipitated with control lgG (Cont) or anti-GFP, and GRAB and Rab8A were detected in the immunoprecipitates (IP) by immunoblotting with anti-GRAB and anti-GFP, respectively. $\boldsymbol{B}$, Ratio of GRAB bound to each Rab8A isoform and normalized to the GRAB to wt Rab8A ratio. Data are mean $\pm S E M ; n=3 .{ }^{*} p<0.05$. C, Association of ca Rab11A with GRAB. GRAB was coexpressed in COS-7 cells with wt Rab11A, ca Rab11A, or ci Rab11A (EGFP-tagged). The cell extracts (input) were immunoprecipitated with control lgG (Cont) or anti-GFP, and GRAB and Rab11A were detected in the immunoprecipitates (IP) by immunoblotting with anti-GRAB and anti-GFP, respectively. D, Quantification of GRAB bound to each Rab11A isoform. Data are mean \pm SEM; $n=3 .{ }^{* *} p<0.01$.

COS-7 cell culture and transfection. COS-7 cells (RCB catalog \#RCB0539, RRID:CVCL_0224) were maintained in DMEM, 10\% (v/v) FBS, $100 \mathrm{U} / \mathrm{ml}$ penicillin, $0.1 \mathrm{mg} / \mathrm{ml}$ streptomycin, and cultures were transfected with plasmids using Lipofectamine 2000 (Invitrogen) or HilyMax (Dojindo) according to the manufacturer's instructions.

Mouse, primary neuron culture and transfection. Pregnant female ICR mice were obtained from Sankyo Laboratory Service. Cdk5-knock-out mice (RRID:IMSR_JAX:003536) were described previously (ContrerasVallejos et al., 2014). All mouse experiments were performed according to the guidelines for animal experimentation of Tokyo Metropolitan University or in compliance with the National Institute of Health's Guidelines on the care and use of laboratory and experimental animals. The studies were approved by the Research Ethics Committee of Tokyo Metropolitan University (approval no. 24-45). All efforts were made to reduce the suffering of animals used. Primary cortical neurons were prepared from mouse brains of either sex at embryonic day 15 or 16 as described previously (Furusawa et al., 2014). Neuron cultures were individually transfected with indicated plasmids using Lipofectamine 2000 at 0 or $2 \mathrm{~d}$ in vitro (DIV) and maintained in neurobasal medium (Invitrogen), 2\% (v/v) B27 (Invitrogen), $0.5 \mu \mathrm{M}$ L-glutamine. To assess GRAB-knockdown efficiency, neurons were nucleofected with each of the pSuper knockdown vectors by electroporation using an Amaxa Nucleofector device (Lonza).

In utero electroporation and tissue processing. Plasmid vectors were injected into lateral ventricle of ICR mouse brain of either sex at embryonic day 14 (E14), as reported previously (Tabata and Nakajima, 2003). For GRAB knockdown, shGRAB SC, \#1, or \#4 (3 $\mu \mathrm{g} / \mu \mathrm{l})$ was coinjected with pCAGGS-EGFP $(1 \mu \mathrm{g} / \mu \mathrm{l})$. For the GRAB overexpression experiments, pEGFP-GRAB wt, $2 \mathrm{~A}$, or $2 \mathrm{D}(5 \mu \mathrm{g} / \mu \mathrm{l})$ was coinjected with pCAGGS-EGFP $(1 \mu \mathrm{g} / \mu \mathrm{l})$. The brains were dissected at E17 and fixed in $4 \%$ PFA $(\mathrm{w} / \mathrm{v})$ overnight at $4^{\circ} \mathrm{C}$ and then changed to PBS containing $30 \%$ sucrose (w/v). Cerebral cortex was sliced into $20 \mu \mathrm{m}$ cortical sections and observed with a confocal laser microscope LSM710 (Carl Zeiss) or macro zoom fluorescence microscope MVX10 (Olympus).

Immunofluorescence staining. COS-7 cells were fixed with $4 \%(\mathrm{w} / \mathrm{v})$ PFA in PBS for 20 min and then treated with $0.1 \%(\mathrm{v} / \mathrm{v})$ Triton X-100 and $5 \%(\mathrm{w} / \mathrm{v})$ skim milk in PBS for permeabilization and blocking. The cells were probed with anti-phospho-Ser 180 in PBS, $0.1 \%(\mathrm{v} / \mathrm{v})$ Triton X-100, $5 \%(\mathrm{w} / \mathrm{v})$ skim milk for $1 \mathrm{~h}$, and then incubated with a secondary antibody and DAPI (Dojindo) in PBS, $0.1 \%(\mathrm{v} / \mathrm{v})$ Triton X-100, 5\% (w/v) skim milk for $1 \mathrm{~h}$.
Primary neurons fixed in $4 \%(\mathrm{w} / \mathrm{v})$ PFA in PBS were treated with PBS, $0.1 \%(\mathrm{v} / \mathrm{v})$ Triton X-100, $5 \%(\mathrm{w} / \mathrm{v})$ normal goat serum for $30 \mathrm{~min}$. The cells were incubated with the primary antibody, anti-pS180, in PBS, $0.1 \%$ $(\mathrm{v} / \mathrm{v})$ Triton X-100, 1\% (w/v) normal goat serum, followed by incubation with a secondary antibody, and were observed using a confocal laser scanning microscope Exciter or LSM710.

Rat hippocampal neurons at 3 DIV were fixed with $4 \%$ (w/v) PFA, $4 \%$ $(\mathrm{w} / \mathrm{v})$ sucrose for $30 \mathrm{~min}$ at $37^{\circ} \mathrm{C}$, permeabilized with $\mathrm{PBS}, 0.1 \%(\mathrm{v} / \mathrm{v})$ Triton X-100 for $5 \mathrm{~min}$, and blocked with 5\% (w/v) BSA, PBS for $1 \mathrm{~h}$. Cells were then incubated with primary antibodies anti-Rab11 (1:150 dilution) or anti-Rab8 (1:100 dilution) in $1 \%(\mathrm{w} / \mathrm{v})$ BSA overnight at $4^{\circ} \mathrm{C}$; anti-rabbit (1:200 dilution) or Alexa-543 anti-mouse (1:500 dilution), for $1 \mathrm{~h}$ at room temperature. Subsequently, the cells were washed with PBS, incubated with streptavidin-Alexa-488 (1:100, Invitrogen) for $30 \mathrm{~min}$, washed, and mounted on slides using FluorSave reagent (Calbiochem). Images were captured using the LSM710 confocal microscope. ImageJ (http://imagej.nih.gov/ij/) was used for colocalization experiments.

Live-cell imaging. COS-7 cells were observed in a Greiner Bio-One glass-bottom dish containing DMEM, 10\% (v/v) FBS. Mouse brain cortical neurons were observed in a polyethylenimine-coated glass-bottom dish containing neurobasal medium supplemented with B27. Fluorescent images were captured every 1 or $2 \mathrm{~s}$ using the LSM710 confocal microscope.

Laemmli SDS-PAGE, Phos-tag SDS-PAGE, and immunoblotting. Laemmli SDS-PAGE and immunoblotting were as described previously (Furusawa et al., 2014). Phos-tag SDS-PAGE gels contained 5\% (w/v) polyacrylamide, $50 \mu \mathrm{M}$ Phos-tag acrylamide (Wako Pure Chemical), and $100 \mu \mathrm{M} \mathrm{MnCl}_{2}$, and electrophoresis was performed as described previously (Kinoshita et al., 2006; Hosokawa et al., 2010).

Immunoprecipitation. COS-7 cells were disrupted in $50 \mathrm{~mm}$ Tris- $\mathrm{HCl}$, pH 7.4, $100 \mathrm{~mm} \mathrm{NaCl}, 1 \mathrm{~mm} \mathrm{MgCl}_{2}, 1$ mм EDTA, 0.5 mм EGTA, 0.5\% (v/v) Nonidet P-40, $0.4 \mathrm{~mm}$ Pefabloc, $10 \mu \mathrm{g} / \mathrm{ml}$ leupeptin, $0.1 \mathrm{~mm}$ DTT, $10 \mathrm{~mm} \mathrm{NaF}$, and $10 \mathrm{~mm} \beta$-glycerophosphate on ice for $10 \mathrm{~min}$. After centrifugation at $17,000 \times g$ for $5 \mathrm{~min}$, each supernatant was incubated with anti-GFP or control IgG for $1 \mathrm{~h}$ on ice and then with Protein $\mathrm{G}$ Sepharose beads (GE Healthcare). After washing four times with the aforementioned buffer, proteins that had been bound to the beads were subjected to SDS-PAGE.

Pull-down of Rab8A by GST-MICAL-L2. Recombinant GST-MICALL2-CC was produced in and purified from Escherichia coli, and then 
A

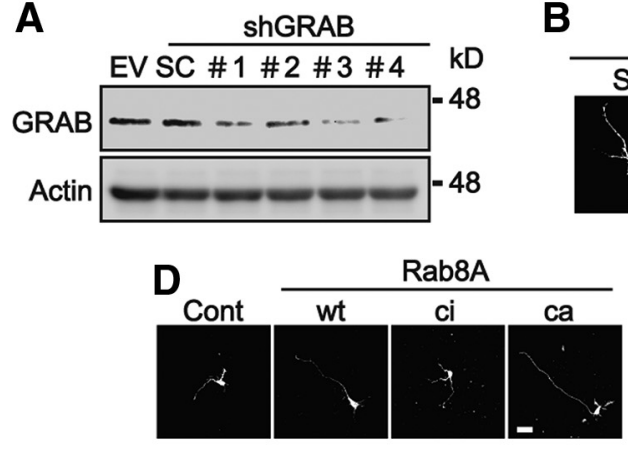

E

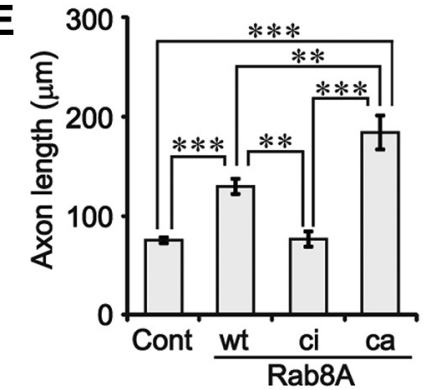

B

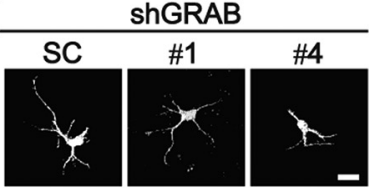

C

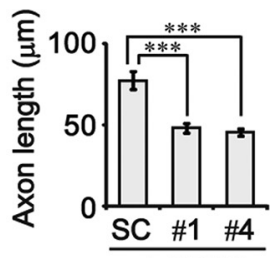

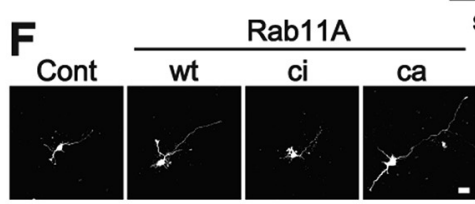

G

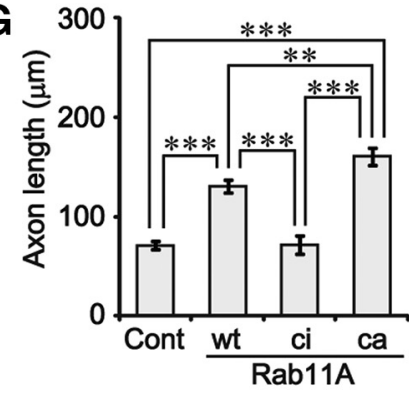

H

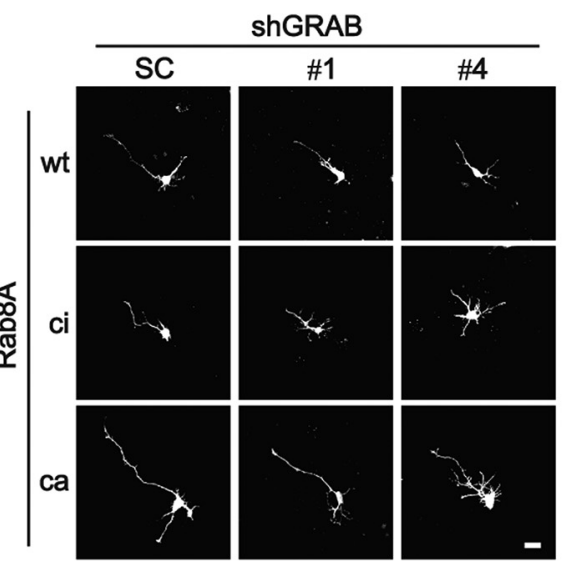

I 200

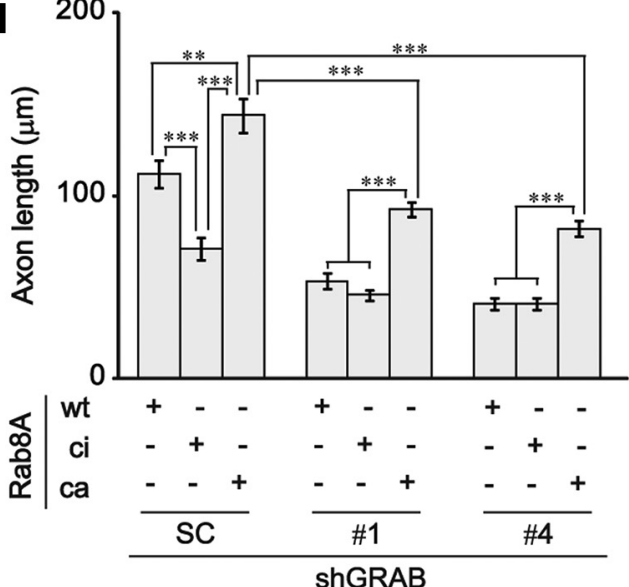

J shGRAB

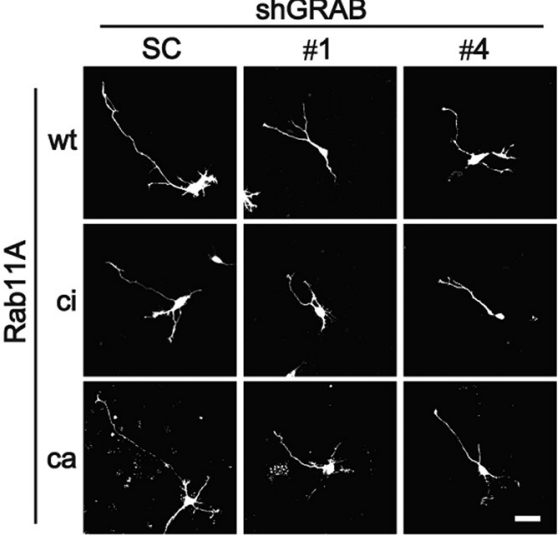

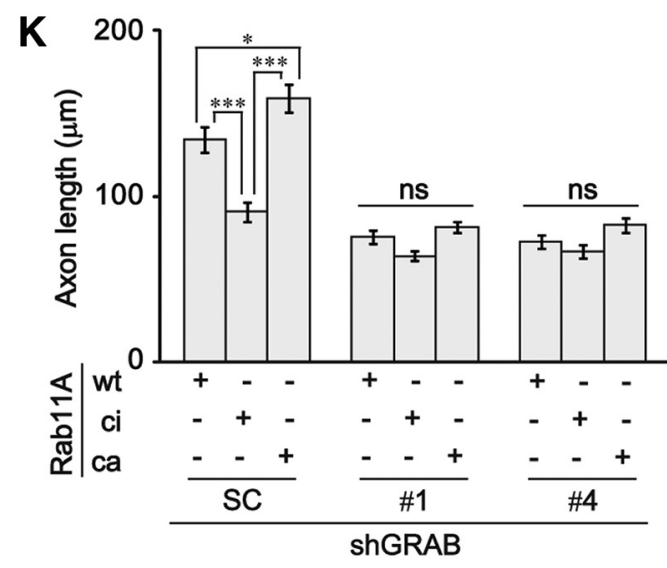

Figure 2. Effect of GRAB knockdown on axon outgrowth. $A, G R A B$ knockdown by shRNAs. Cortical neurons were transfected with shGRAB \#1-4, SC, or an empty vector (EV) at 0 DIV, and cell lysates were immunoblotted with anti-GRAB at 3 DIV. Actin is the control. $B$, Effect of GRAB knockdown on axon outgrowth. Cortical neurons were transfected with SC, shGRAB \#1, or \#4 with EGFP for visualization at 0 DIV, and axon length was measured at 3 DIV. Scale bar, $20 \mu \mathrm{m}$. C, Axon lengths of neurons expressing EGFP. Data are mean \pm SEM. SC, $n=56$; shGRAB \#1, $n=62 ;$ shGRAB $\# 4, n=60$. D, Effect of Rab8A overexpression on axonal outgrowth in primary neurons. EGFP (Cont), EGFP-Rab8A wt, ci (T22N), or ca (Q67L) was individually transfected into mouse brain cortical neurons at 0 DIV, and axonal outgrowth was observed by fluorescence of EGFP at 3 DIV. Scale bar, $20 \mu \mathrm{m}$. $E$, Quantification of axon length in EGFP-expressing neurons. Data are mean \pm SEM; $n=$ 71 for Cont, $n=75$ for Rab8A wt, $n=70$ for Rab8A ci, and $n=67$ for Rab8A ca. $F$, Effect of Rab11A overexpression on axonal outgrowth in primary neurons. EGFP (Cont), EGFP-Rab11A wt, ci (S25N), or ca (Q70L) was individually transfected into mouse brain cortical neurons at 0 DIV, and axonal outgrowth was observed by fluorescence of EGFP at 3 DIV. Scale bar, $20 \mu \mathrm{m}$. G, Quantification of axon length in EGFP-expressing neurons. Data are mean \pm SEM; $n=60$ for each of Cont, Rab11A wt, Rab11A ci, and Rab11A ca. $\boldsymbol{H}$, Effect of GRAB knockdown on Rab8A-dependent axonal outgrowth. Cortical neurons were cotransfected with shGRAB \#1, \#4, or SC, and wt Rab8A, ci Rab8A, or ca Rab8A (EGFP-tagged) at 0 DIV, and axon length was measured at 3 DIV. Scale bar, $20 \mu \mathrm{m}$. I, Axon lengths for neurons expressing EGFP. Data are mean \pm SEM. SC and wt Rab8A, ci Rab8A, or ca Rab8A, $n=51, n=54$, and $n=50$, respectively; shGRAB \#1 (Figure legend continues.) 
bound to Glutathione-Sepharose beads (GE Healthcare) (Sano et al., 2008). The beads were incubated with COS-7 cell extracts that had expressed wt EGFP-Rab8A and wt EGFP-GRAB, EGFP-GRAB 2A, or EGFP-GRAB 2D. After washing with $50 \mathrm{~mm}$ Tris-HCl, pH 7.4, $150 \mathrm{~mm}$ $\mathrm{NaCl}, 1 \mathrm{~mm} \mathrm{MgCl}, 0.2 \%$ (v/v) Triton X-100, 0.4 mм Pefabloc, $10 \mu \mathrm{g} / \mathrm{ml}$ leupeptin, $0.1 \mathrm{~mm}$ DTT, $10 \mathrm{~mm} \mathrm{NaF}$, and $10 \mathrm{~mm} \beta$-glycerophosphate, proteins bound to the beads were subjected to immunoblotting with anti-EGFP to assess the presence of active EGFP-Rab8A.

Quantification and statistical analysis. Immunoreactions were visualized by exposing the blots to $\mathrm{x}$-ray film or acquiring a digital image with a Fusion SL4 imager (Vilber Lourmat). Band intensities were quantified using ImageJ (RRID:SCR_003070). The longest neurite of the primary neurons at 3 DIV was defined as the axon, and its length was measured using ZEN imaging software (ZEN Digital Imaging for Light Microscopy, RRID:SCR_013672) (Carl Zeiss). The length of the leading process was measured using ImageJ. Total GRAB and the ratio of phosphorylated GRAB to total GRAB were obtained by quantifying fluorescence intensity in the whole-cell body, a part of axon and the whole growth cone using ZEN imaging software. Quantitative data are expressed as the mean \pm SEM and were subjected to the Student's $t$ test for single comparisons or one-way ANOVA analysis, followed by Tukey's multiple-comparisons test for multiple comparisons.

\section{Results}

GRAB interacts preferably with constitutively inactive (ci) Rab8A and constitutively active (ca) Rab11A

GRAB was originally reported to be a GEF for Rab3A (Luo et al., 2001), but recent reports indicate that it is a GEF for Rab8 instead (Yoshimura et al., 2010; Guo et al., 2013) and bind Rab11 (Horgan et al., 2013). We first confirmed that GRAB interacted with Rab8 and Rab11 using coimmunoprecipitation. When wt Rab8A, ca Rab8A (Q67L mutant), or ci Rab8A (T22N mutant) was coexpressed with GRAB, a larger amount of GRAB was detected in the immunoprecipitate of ci Rab8A, compared with wt or ca Rab8A (Fig. $1 A, B$ ). When GRAB was coexpressed with wt Rab11A, ca Rab11A (Q70L mutant), or ci Rab11A (S25N mutant), and immunoprecipitated with Rab11A, a larger amount of GRAB coprecipitated with ca Rab11A than with wt or ci Rab11A (Fig. 1C,D). These results confirmed that GRAB is an effector of Rab11A (Horgan et al., 2013).

\section{GRAB regulates axon outgrowth upstream of Rab8A and downstream of Rab11A}

Because nothing is known concerning a role for GRAB during axonal outgrowth, we assessed its possible role by knocking down GRAB expression in cultured neurons. We prepared four different shRNA sequences for GRAB, shGRAB \#1-4, and found that shGRAB \#1, \#3, and \#4 decreased GRAB expression (Fig. 2A). shGRAB \# 1 and \#4 were then used to evaluate the role of GRAB in axon outgrowth. Knockdown by either shRNA decreased axonal lengths (Fig. $2 B, C$ ), indicating the involvement of GRAB in axon outgrowth.

Possibly GRAB promotes axon outgrowth via activation of Rab8, although only one report has investigated the role of Rab8 in neurite outgrowth (Huber et al., 1995). We therefore examined whether Rab8A was involved in axon outgrowth by overex-

(Figure legend continued.) and wt Rab8A, ci Rab8A, or ca Rab8A, $n=59, n=58$, and $n=55$, respectively; shGRAB \#4 and wt Rab8A, ci Rab8A, or ca Rab8A, $n=52, n=51$, and $n=51$, respectively. J, Effect of GRAB knockdown on Rab11A-dependent axonal outgrowth. Cortical neurons were cotransfected with SC, shGRAB \#1, or \#4 and wt Rab11A, ci Rab11A, or ca Rab11A (EGFP-tagged) at 0 DIV, and axon length was measured at 3 DIV. Scale bar, $20 \mu \mathrm{m}$. $\boldsymbol{K}$, Axon lengths of neurons expressing EGFP. Data are mean \pm SEM. Each set of an shRNA and a Rab11A isoform; $n=60 .{ }^{*} p<0.05 .{ }^{* *} p<0.01 .{ }^{* * *} p<0.001$. pressing its isoforms in mouse cortical neurons. Wild-type Rab8A, ca Rab8A, and ci Rab8A, each as an EGFP construct, were individually transfected into cultured neurons at 0 DIV, and axonal length was measured at 3 DIV. Transfected wt Rab8A increased axonal outgrowth by $\sim 60 \%$. ca Rab8A enhanced it more than twofold, but ci Rab8A did not enhance outgrowth compared with the other two isoforms (Fig. 2D,E). Therefore, Rab8A has a positive role in axonal elongation. To confirm the role of Rab11 in axon outgrowth, we overexpressed wt Rab11A, ca Rab11A, or ci Rab11A in mouse cortical neurons. wt Rab11A or ca Rab11A overexpression induced approximately a twofold increase in axonal length, whereas ci Rab11A did not (Fig. 2F, G), consistent with our previous report (Takano et al., 2012).

We next asked whether GRAB acts upstream or downstream of Rab8A. GRAB was knocked down in the neurons in the presence of transfected wt, ci, or ca Rab8A. GRAB knockdown reduced axon length regardless of which Rab8A construct was expressed. In particular, the axon lengths of neurons expressing wt Rab8A were similar to that of ci Rab8A-expressing neurons. However, neurons expressing ca Rab8A had longer axons than those expressing wt or ci Rab8A, indicating that GRAB acts on axon growth upstream of Rab8A. Nonetheless, ca Rab8A did not reverse the knockdown effect of GRAB to that of control scramble (CA) shRNA (Fig. $2 H, I$ ), which suggests that, in addition to the Rab8A pathway, a second GRAB-related pathway for axon outgrowth exists.

We showed, as described above, that GRAB interacts preferably with the ca form of Rab11A (Fig. 1C,D). To determine whether GRAB is an effector of Rab11A in axon outgrowth, we knocked down GRAB with shGRAB \#1 or \#4 in cultured cortical neurons expressing wt, ca, or ci Rab11A (Fig. $2 \mathrm{~J}, K$ ). In control experiments in the presence of SC shRNA, ca Rab11A enhanced axon outgrowth and ci Rab11A suppressed it (Fig. 2 J,K). These results are consistent with those for which an shRNA was absence (Takano et al., 2012). GRAB knockdown decreased the axon length of neurons cotransfected with all Rab11A isoforms (Fig. $2 J, K)$, which is unlike that found for the Rab8A isoforms. These results suggest that $\mathrm{GRAB}$ acts downstream of Rab11A during axon elongation.

\section{Cdk5-p35 phosphorylates GRAB at Ser169 and Ser180}

We next searched for phosphorylation sites on GRAB that might be involved in its regulation. We identified three possible Cdk5phosphorylation sites at Ser169, Ser180, and Thr213, which are near the GEF domain of GRAB (Fig. 3A). We expressed GRAB with Cdk5 and p 35 in COS-7 cells to determine the phosphorylation state, which was assessed using Phos-tag SDS-PAGE for which phosphorylated proteins have markedly decreased mobilities compared with those found for conventional SDS-PAGE (Kinoshita et al., 2006; Hosokawa et al., 2010). GRAB migrated as a single band in an SDS-PAGE gel whether or not it was expressed with Cdk5-p35 (Fig. 3B, bottom). In a Phos-tag SDS-PAGE gel, GRAB appeared as multiple bands even when expressed alone (Fig. $3 B$, top), suggesting that GRAB had been phosphorylated by an endogenous kinase(s). When coexpressed with active Cdk5p35, but not when expressed with a kinase negative $(\mathrm{kn}) \mathrm{Cdk} 5$ p35, all GRAB molecules migrated more slowly and as a single band (Fig. 3B, top), indicating that it had been phosphorylated by Cdk5-p35.

To identify the GRAB phosphorylation sites, we mutated Ser169, Ser180, or Thr213 in GRAB to an Ala to form S169A, S180A, and T213A, and double Ala mutant of S169/180A. These constructs were expressed individually in the presence or absence 
A

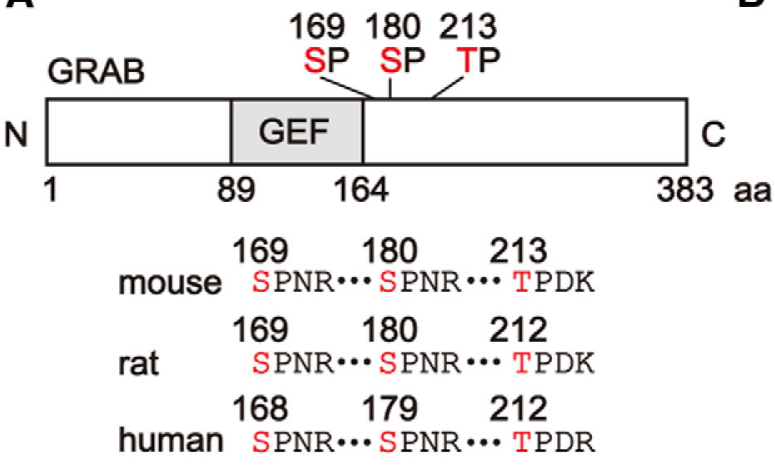

B

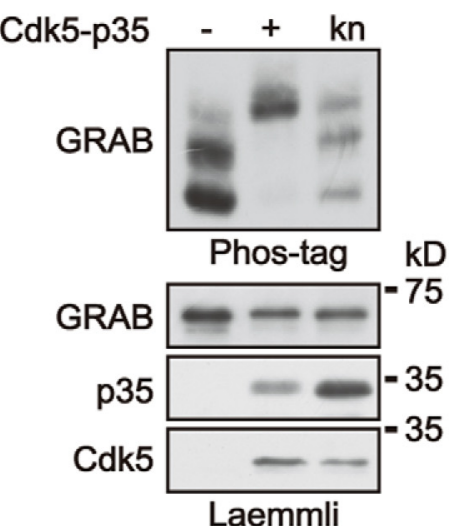

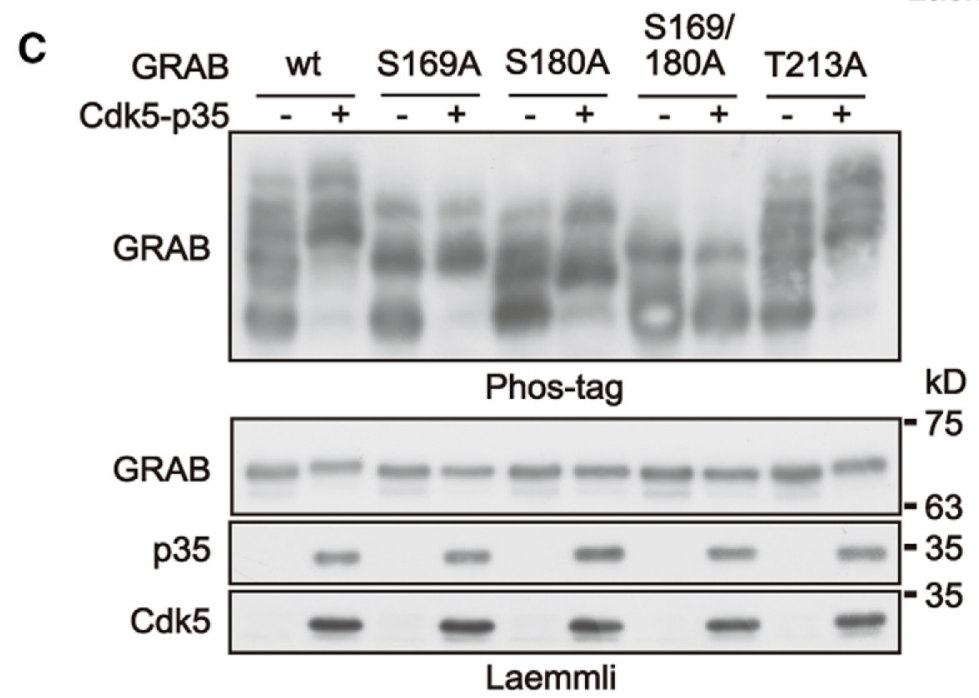

D

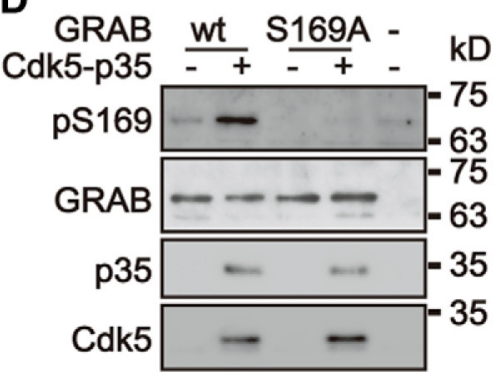

E

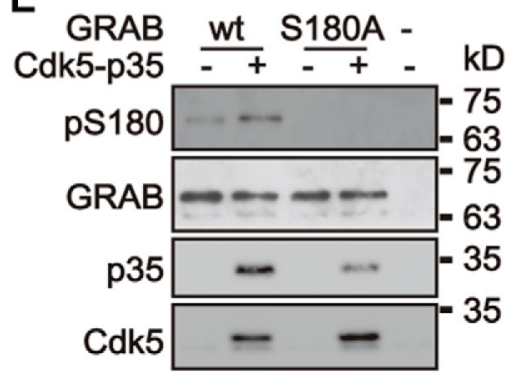

$\mathbf{F}$
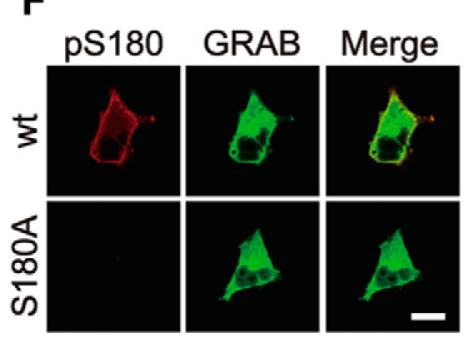

G
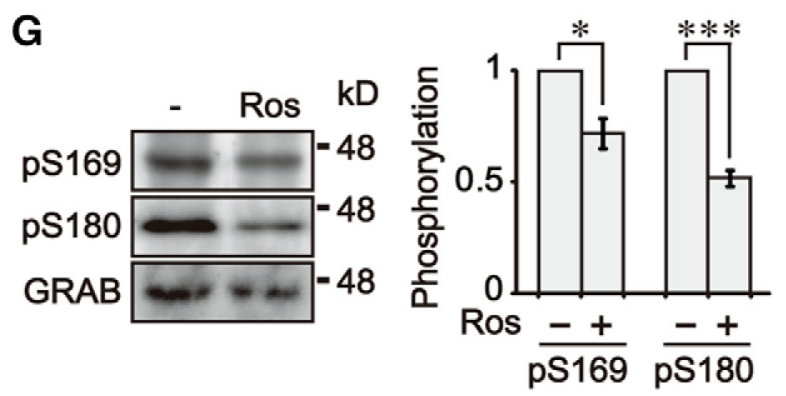

Figure 3. Cdk5-p35 phosphorylates GRAB at Ser169 and Ser180.A, Schematic of the GRAB molecule showing the GEF domain and three possible Cdk5-phosphorylation sites, which are conserved in mouse, rat, and human GRAB. B, Phosphorylation of GRAB by Cdk5-p35. EGFP-GRAB was cotransfected with Cdk5-p35 (+) or a kinase negative Cdk5-p35 (kn) into C0S-7 cells. Cell lysates were subjected to Laemmli (bottom) or Phos-tag SDS-PAGE (top) followed by immunoblotting as indicated. C, Phosphorylation of GRAB at Ser169 and Ser180 by Cdk5-p35. wt GRAB, GRAB S169A, GRAB S180A, GRAB S169/180A, or GRAB T213A (all EGFP constructs) was expressed with $(+)$ or without $(-)$ Cdk5-p35. Immunoblotting was performed as indicated after Laemmli (bottom) or Phos-tag SDS-PAGE (top). D, E, EGFP-GRAB wt or S169A (D) or S180A (E) was coexpressed with or without Cdk5-p35 in COS-7 cells. The cell lysates were blotted with anti-pS169 (D) or anti-pS180 (E), and with anti-GFP (GRAB), anti-p35, and anti-Cdk5.F, EGFP-GRAB wt (top) or S180A (bottom) was coexpressed with (dk5-p35, and cells were stained with anti-pS180. GRAB is shown with EGFP. $G$, Phosphorylation of GRAB at Ser 169 and Ser 180 in neurons. Primary cortical neurons at 5 DIV were treated with the Cdk5 inhibitor roscovitine (Ros) for $4 \mathrm{~h}$. Cell lysates were then immunoblotted with anti-pS169, anti-pS180, and anti-GRAB. Quantification is shown on right. ${ }^{*} p<0.05 .{ }^{* *} p<0.001$. 
of Cdk5-p35, and their Phos-tag SDS-PAGE migration patterns were examined. The migration rates seen for S169A and S180A, and $S 169 / 180 A$ were increased in comparison with wt GRAB in the Phos-tag SDS-PAGE gel, and the upper bands were completely absent for S169/180A (Fig. 3C). Conversely, the migration rates for T213A were mostly unaffected by the absence or presence of Cdk5-p35. These results clearly indicate that GRAB Ser169 and Ser180 are Cdk5-phosphorylation sites.

To confirm the in vivo phosphorylation of GRAB, we individually generated antibodies against GRAB phospho-Ser169 (anti-pS169) and phospho-Ser180 (anti-pS180). Anti-pS169 and anti-pS180 reacted strongly with wt GRAB, but not with S169A or S180A, respectively, when coexpressed with Cdk5-p35 (Fig. $3 D, E)$. Weak detection of wt GRAB in the absence of Cdk5-p35 suggested that the sites were phosphorylated to a certain extent by an endogenous kinase(s). The specificity of pS180 for immunofluorescence staining was tested by expression of each GRAB isoform with Cdk5-p35 in COS-7 cells. Anti-pS180 stained cells expressing wt GRAB but not GRAB 2A-expressing cells (Fig. $3 F$ ). Although total GRAB, observed as EGFP, was found evenly throughout the cytoplasm, anti-pS180 staining was detected more strongly at the cell periphery (Fig. $3 F$ ). (We could not use anti-pS169 for immunostaining because the antibody lost activity upon purification.)

Phosphorylation at Ser169 and Ser180 by Cdk5 was confirmed for primary neurons using the antibodies. Endogenous GRAB migrated at $\sim 48 \mathrm{kDa}$ in an SDS-PAGE gel and reacted on blots with these antibodies, indicating that phosphorylation of GRAB had occurred in the neurons. When the neurons were treated with the Cdk5 inhibitor, roscovitine, the amounts of phosphorylated GRAB S169 and S180 was reduced to $72 \pm 7 \%$ and $52 \pm 4 \%$, respectively (Fig. $3 G$ ), indicating the phosphorylation of GRAB at Ser 169 and Ser 180 by Cdk5 in neurons.

\section{Phosphorylation of GRAB regulates axonal outgrowth via Rab8A}

We next investigated whether the axon outgrowth activity of GRAB is regulated by its phosphorylated state. Axonal length was measured in primary neurons expressing wt GRAB, its nonphosphorylatable mutant GRAB 2A (S169/180A), or the phosphomimetic mutant, GRAB 2D (S169/180D). Wild-type GRAB and 2D mutant increased the axonal length 1.7- and 1.5-fold, respectively, compared with control neurons, which had been transfected with only EGFP (Fig. 4A,B). GRAB 2A increased axonal length 2.4-fold compared with control neurons. Thus, when not phosphorylated (i.e., in the form of GRAB 2A), GRAB more strongly increased axon outgrowth than when phosphorylated (i.e., in the form of GRAB 2D).

Next, we tested whether the phosphorylation of GRAB occurs upstream of Rab8A by coexpressing GRAB wt, GRAB 2A, or GRAB 2D with wt, ca, or ci Rab8A. Neurons expressing GRAB 2A had longer axons than those expressing GRAB 2D when coexpressed with wt Rab8A (Fig. 4C,D) as was observed when GRAB $2 \mathrm{~A}$ or $2 \mathrm{D}$ was expressed alone (Fig. $4 A, B$ ). However, a difference in axon length for GRAB $2 A$ and GRAB $2 D$ was absent when they were coexpressed with ca or ci Rab8A, with longer neuronal axons found when ca Rab8A was expressed compared with ci Rab8A expression (Fig. 4C,D). Thus, mutations of GRAB Ser169 and Ser180 to Ala or Asp did not impact the effect of ca or ci Rab8A on axon elongation. These results also confirm that GRAB regulates axonal outgrowth upstream of Rab8A.

\section{Phosphorylation of GRAB regulates its GEF activity but does not affect its interaction with Rab11A}

GRAB Ser169 and Ser180 are located downstream of its GEF domain (Fig. 3A). We investigated the effect of phosphorylation on its GEF activity for Rab8A using MICAL-L2, an effector of Rab8 (Fukuda et al., 2008; Yamamura et al., 2008). We expressed wt GRAB, GRAB 2A, or GRAB 2D, and wt Rab8A in COS-7 cells and quantified the levels of active Rab8A bound to the GSTMICAL-L2-CC-beads. A larger amount of Rab8A was pulled down when it was coexpressed with GRAB $2 A$ than with wt GRAB or GRAB 2D (Fig. 5A,B), indicating that Cdk5-p35 phosphorylation of GRAB suppresses its GEF activity for Rab8A.

GRAB has a Rab11A-binding site in its C-terminal region, which contains the essential sequence, ILFAEF, at positions 223 228 (Horgan et al., 2013). We wondered whether phosphorylation at S169 and/or S180 affects association of GRAB and Rab11A. To answer this question, we cotransfected wt GRAB, 2A or 2D, with wt Rab11A and assessed their binding by immunoprecipitation. Wild-type GRAB, GRAB 2A, and GRAB 2D bound wt Rab11A to a similar extent (Fig. $5 C, D$ ). To evaluate whether GRAB isoforms have a preferential binding to ca Rab11A, as expected for a small GTPase effector protein, we immunoprecipitated ca Rab11A in the presence of wt GRAB, GRAB 2A, or GRAB 2D. We observed that GRAB binding to ca Rab11A was similar to wt Rab11A (Fig. 5E, F). Therefore, we concluded that GRAB binds to Rab11A, irrespective of its phosphorylation status.

\section{Phosphorylation of GRAB regulates its recruitment of Rab8A to Rab11A-positive endosomes}

Certain GEFs activate their target Rabs and then recruit them to a specific membrane compartment (Blümer et al., 2013). We asked whether phosphorylation of GRAB affected its association with Rab8A by coexpressing wt Rab8A with wt GRAB, GRAB 2A, or GRAB 2D and assessing whether the GRAB isoforms had bound Rab8A in the Rab8A immunoprecipitates. GRAB 2A preferentially bound to wt Rab8A, whereas GRAB 2D showed a decreased interaction (Fig. 6A,B). Wild-type GRAB showed no significant differences from GRAB 2A, likely because Cdk5 is not active in COS-7 cells, leading to a higher ratio of unphosphorylated GRAB. On the other hand, GRAB 2D might mimic the phosphorylated form only partially, probably because of its less minus charge. Considering that GEF binds to ci Rab, we performed the binding experiment using ci Rab8A (Fig. $6 C, D$ ). The results were similar to those of wt Rab8A, indicating that the substantial amount of wt Rab8A is present as the inactive form.

Concerning endocytic Rab cascades, a coupling effect between two different Rabs is found such that a Rab effector for one $\mathrm{Rab}$ is a GEF for a second Rab (Hutagalung and Novick, 2011). The aforementioned results suggested that GRAB mediates the activation and recruitment of Rab8A- to Rab11A-positive endosomes, with the recruitment being regulated by Cdk 5 phosphorylation of GRAB. When we coexpressed EGFP-Rab8A and mStr-Rab11A in the absence of GRAB transfection, the Rabs were mostly found in different vesicles, as evidenced by the near absence of yellow vesicles (Fig. 6E, left column). In the peripheral cytoplasm, most of the vesicles were labeled by Rab8A or Rab11A, but not by both. At the perinuclear region, both Rabs accumulated, but not to a great extent, in the same vesicle. When wt GRAB was transfected with Rab8A and Rab11A, the colocalization of the two Rabs was $\sim 76 \%$, a threefold increase over that found in the absence of transfected GRAB (Fig. $6 F$ ).

Recruitment of EGFP-Rab8A to mStr-Rab11A-positive vesicles was also observed by dual-color live-cell imaging in the 
A

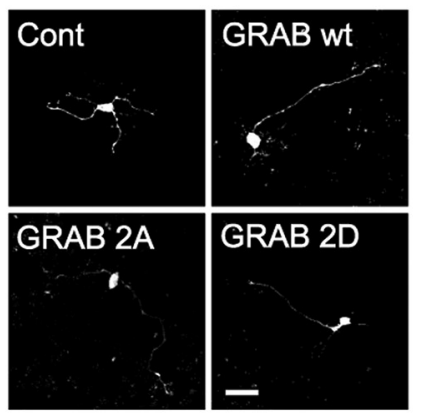

B

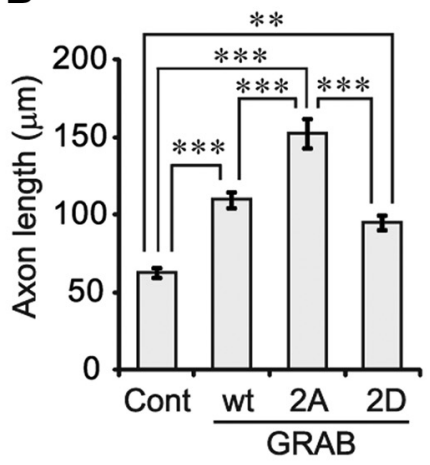

C

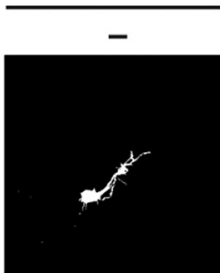

GRAB
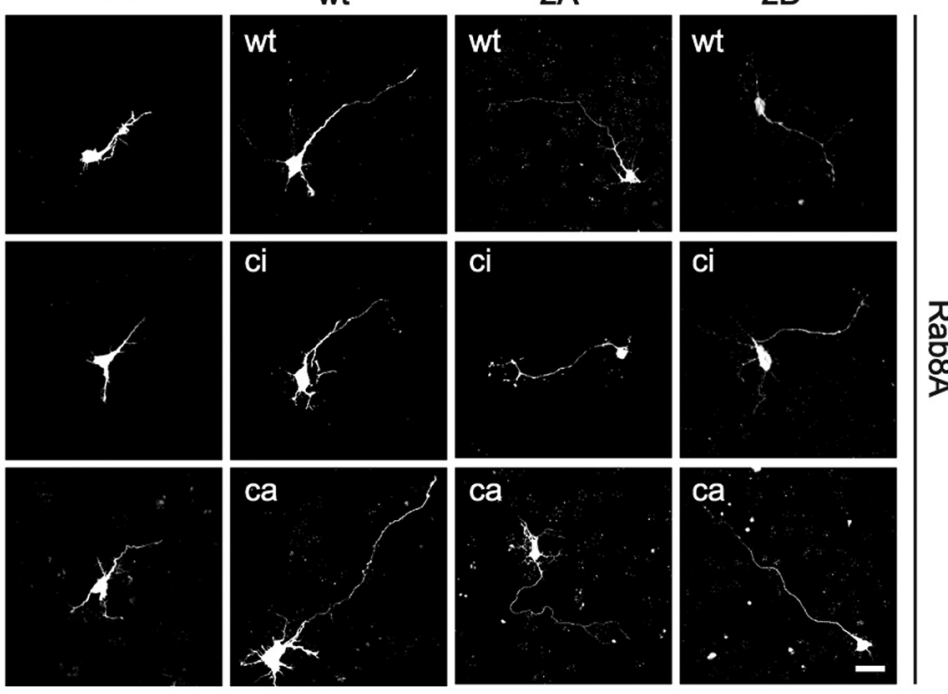



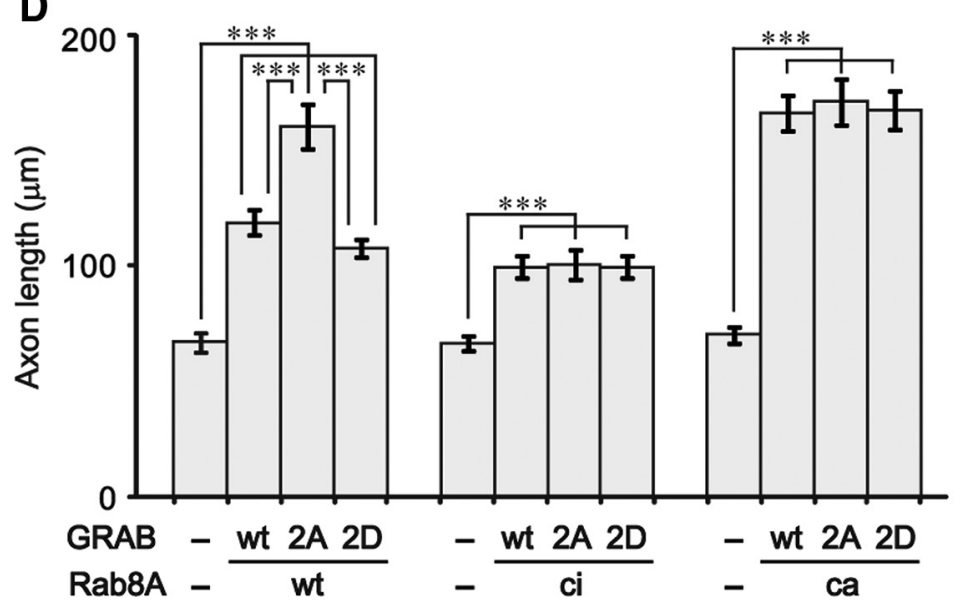

Figure 4. Effect of phosphorylation of $G R A B$ by $(d k 5$ on axon outgrowth. $A$, Effect of GRAB overexpression on axonal outgrowth in primary neurons. EGFP (Cont), wt GRAB, GRAB 2A (S169/180A), or GRAB 2D (S169/180D) (EGFP-tagged) was individually transfected into cortical neurons at 0 DIV, and axonal outgrowth was observed by EGFP fluorescence at 3 DIV. Scale bar, $20 \mu \mathrm{m}$. $B$, Quantification of axonal lengths in EGFP-expressing neurons. Data are mean \pm SEM. Cont, $n=57$; wt GRAB, $n=66$; GRAB 2A, $n=68 ;$ GRAB 2D, $n=53$. C, Effect of phosphorylation of GRAB on Rab8A-stimulated axonal outgrowth. wt Rab8A, ci Rab8A, or ca Rab8A (EGFP-tagged) was individually cotransfected with GRAB wt, 2A, or 2D into cortical neurons at 0 DIV, and axonal outgrowth was observed by EGFP fluorescence at 3 DIV. Left panels, Control neurons expressing EGFP alone ( - ). Scale bar, $20 \mu \mathrm{m}$. D, GRAB regulates axonal outgrowth upstream of Rab8A. Quantification of axonal length in EGFP-expressing neurons. Data are mean \pm SEM. wt Rab8A and GRAB wt, GRAB 2A, or GRAB 2D, $n=60, n=61$, or $n=65$, respectively; ci Rab8A and GRAB wt, GRAB 2A, or GRAB 2D, $n=60, n=66$, or $n=62$, respectively; ca Rab8A and GRAB wt, GRAB 2A, or GRAB 2D, $n=60, n=60$, or $n=61$, respectively. Control was taken in a respective set of experiments $\left(n=60\right.$ for each). ${ }^{* *} p<0.01 .{ }^{* *} p<0.001$. presence of transfected wt GRAB. A gradual accumulation of EGFP-Rab8A on mStrRab11A-positive endosomes was observed with only a slight change in mStr fluorescence intensity during that time (Fig. $6 G, H)$. Conversely, we did not observe accumulation of Rab11A on Rab8A-positive endosomes. We hypothesized that this recruitment is regulated by phosphorylation of GRAB. To test this hypothesis, we characterized the colocalization of Rab8A and Rab11A in the presence of GRAB 2A or GRAB 2D. In the presence of GRAB 2A, colocalization was $88 \%$ (Fig. 6E,F, “2A"), whereas colocalization was only $43 \%$ for GRAB $2 \mathrm{D}$ (Fig. $6 E, F$, “2D”), indicating that, when phosphorylated, GRAB has a decreased ability to recruit Rab8A onto Rab11Apositive endosomes.

\section{Association of GRAB with Rab8A and}

Rab11A-positive endosomes in neurons Given that phosphorylation suppressed the GEF and recruiting activities of GRAB for Rab8A, we next evaluated the subcellular location(s) of phosphorylated GRAB in neurons. Cultured neurons were then transfected with mStr-GRAB and the location of phosphorylated GRAB was observed by immunostaining with antipS180. Total GRAB, observed as mStr, was found throughout the cell-body cytoplasm (Fig. 7A). Anti-pS180 stained cytoplasmic vesicles but more strongly stained those at the cytoplasm periphery (Fig. $7 A$ ). The axon (Fig. 7B) and growth cone (Fig. 7C) vesicles that $\mathrm{mStr}$ fluoresced were also usually stained by anti-pS180. The ratio of total and phosphorylated GRAB was measured in the above three regions of neurons and shown in Figure 7D. Phospho-GRAB was increased in the axon and growth cone compared with that in the cell body (Fig. 7D; pS180/ GRAB). This result suggests that phosphorylated GRAB is selectively transported from the cell body to the growth cone through the axon or GRAB is phosphorylated during transportation in the axon. Nevertheless, the amount of dephosphorylated GRAB appeared to increase in the growth cone because the total GRAB was more abundant in the growth cone than the axon (Fig. 7D; GRAB).

Endogenous Rab11 accumulated at the perinuclear region of rat hippocampal neurons, whereas Rab8 was more diffusely distributed in the cell body (Fig. $7 E)$. We found that these endogenous Rab8 and Rab11 partially colocalized in cell bodies (Pearson's colocalization coefficient, 0.30) and weakly in axons (Pear- 

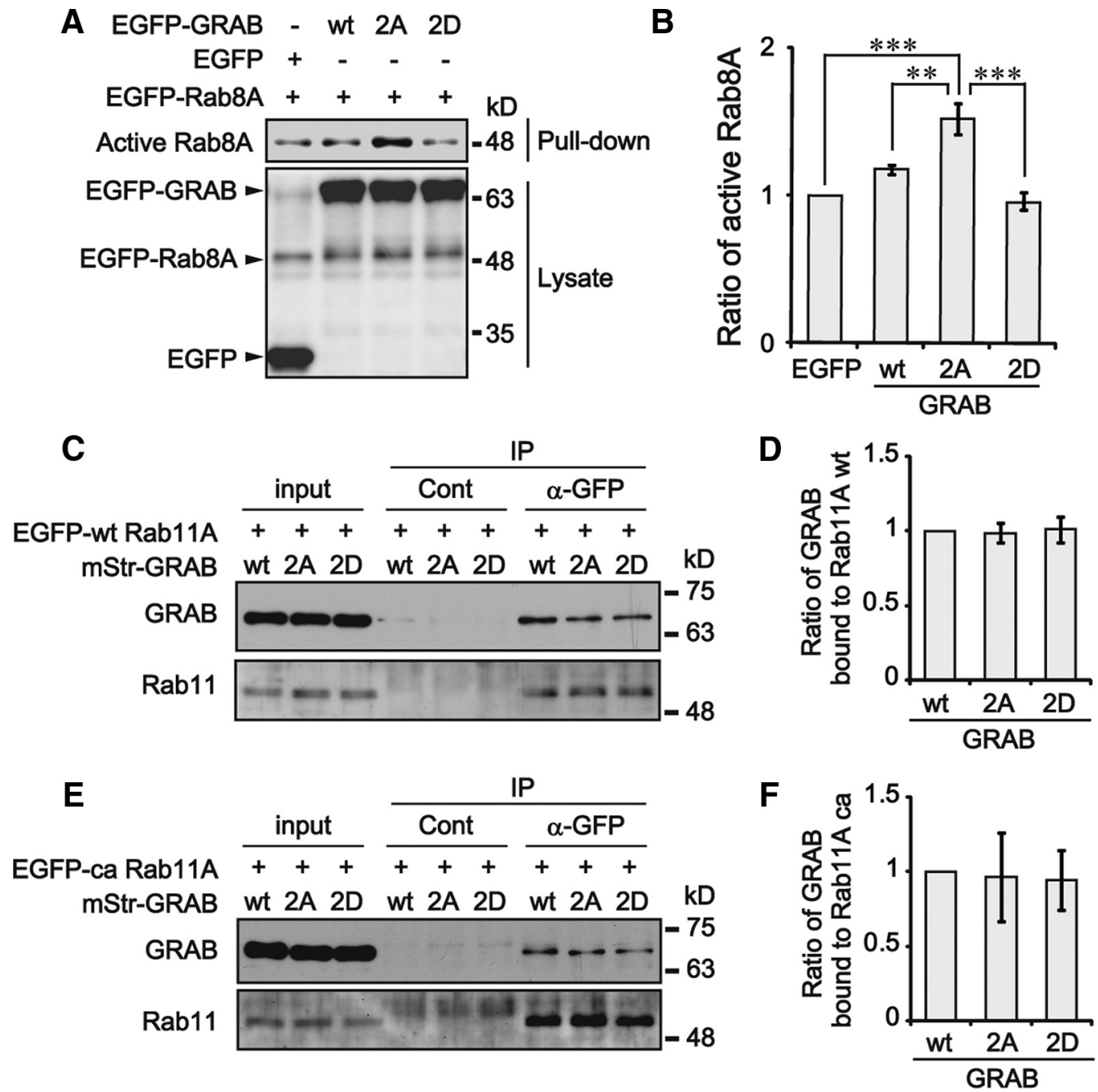

Figure 5. Effect of phosphorylation of GRAB on its GEF activity and interaction with Rab11A. A, Effect of phosphorylation on GRAB GEF activity. COS-7 cells were cotransfected with Rab8A and wt GRAB, GRAB 2A, or GRAB 2D (EGFP-tagged), and active Rab8A was pulled down by GST-MICAL-L2-CC. Bottom, Cell lysate. Top, Rab8A pulled down. $B$, Quantification of active Rab8A. Relative ratios to the EGFP-expressing cells are expressed as mean \pm SEM; $n=4 .{ }^{* *} p<0.01 .{ }^{* * *} p<0.001$. C, E, Effect of mutated Cdk5-phosphorylation sites in GRAB in association with Rab11A. wt GRAB, GRAB 2A, or GRAB 2D (all mStr constructs) was coexpressed in COS-7 cells with EGFP-wt Rab11A (C) or EGFP-ca Rab11A (E). Rab11A was immunoprecipitated with anti-GFP, and GRAB and Rab11 were detected in the immunoprecipitates by immunoblotting with anti-GRAB and anti-EGFP, respectively. $\boldsymbol{D}, \boldsymbol{F}$, Relative amount of each GRAB isoform bound to wt Rab11A (D) or ca Rab11A $(\boldsymbol{F})$ and normalized to wt GRAB. Data are mean \pm SEM. Each GRAB isoform; $n=3$.

son's colocalization coefficient 0.08; Fig. $7 F$ ). As shown above, Cdk5 phosphorylation of GRAB decreased its interaction with Rab8A but not with ca Rab11A Q70L (Figs. 5C,D, 6A, B), suggesting that Rab11A-positive vesicles bind phosphorylated GRAB. To identify the location of phosphorylated GRAB, we transfected mStr-GRAB with EGFP-Rab8A or EGFP-Rab11A, and then immunostained those neurons with anti-pS180. In the cell body, anti-pS180 stained vesicles labeled with Rab11A but not with Rab8A (Fig. 7G, bottom right, arrows). This dotted-type staining was found in axons and growth cones. In the axon, most GRABpositive vesicles were also stained with anti-pS180, and those vesicles were also labeled with Rab11A (Fig. $7 H$, bottom right) but not with Rab8A (Fig. $7 \mathrm{H}$, bottom left). There were a small number of GRAB-positive and Rab8A-positive vesicles in the axons, but they were not stained with anti-pS180 (Fig. $7 H$, bottom left, arrowhead). Thus, Rab8A associated primarily with vesicles with nonphosphorylated GRAB in neurons. Two vesicles containing Rab8A and GRAB are shown in growth cone (Fig. 7I, left panels). Anti-pS180 stained them differently. These vesicles might represent a transition involving phosphorylated to de- phosphorylated GRAB, which can activate and associate with Rab8A. Expression of Rab11A was mainly found in the shafts of axon, which was also strongly labeled with phosphorylated GRAB and total GRAB (Fig. 7I, right panels). It appears, therefore, that phosphorylated GRAB is conveyed on endosomes to the tip of the axon by active Rab11A.

GRAB is transported on Rab11A-positive endosomes in axons The aforementioned results suggested that phosphorylated GRAB is transported on Rab11A-positive endosomes in the axon to the growth cone. We observed the movements of EGFP-ca Rab11A and mStr-GRAB in axons by live-cell imaging (Fig. $8 A$ ). An example is shown in Figure $8 A$, where three vesicles are only ca Rab11A-labeled and the other three are GRAB/ca Rab11A double-labeled. Thus, GRAB-positive vesicles also contain Rab11A, and GRAB in these vesicles would be phosphorylated as shown by our immunostaining data. Several patterns of the movement were observed (Fig. $8 A$ ). Although one doubly labeled vesicle did not move during the $32 \mathrm{~s}$ observation period (Fig. $8 \mathrm{~A}$, asterisk), the other two GRAB/ca Rab11A-double-positive vesi- 
A

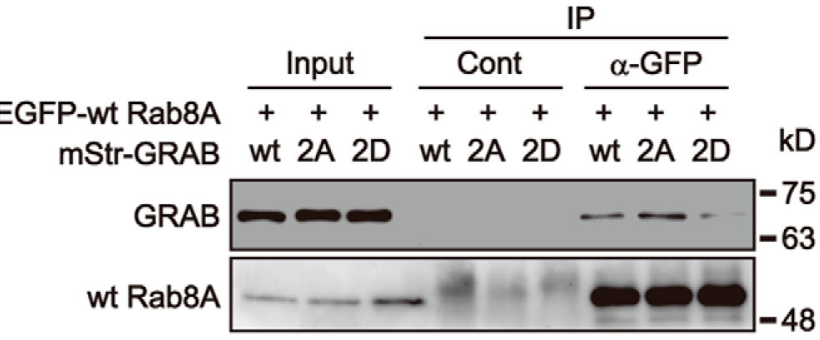

C

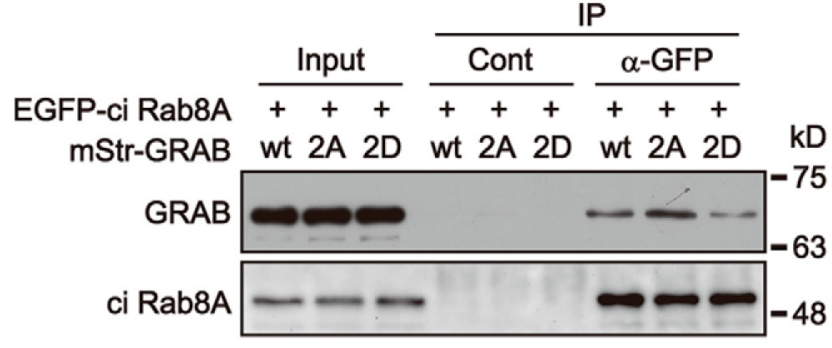

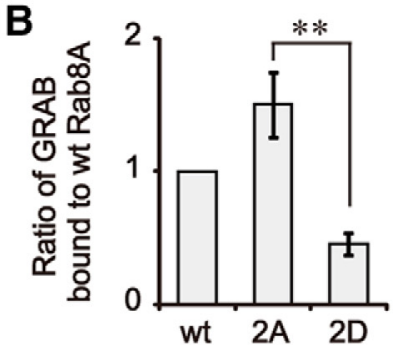

D

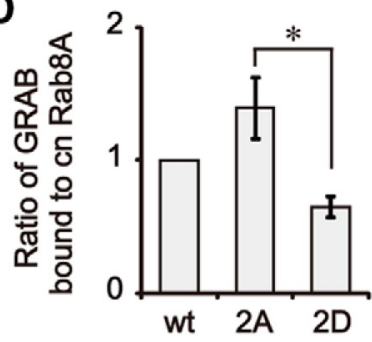

E
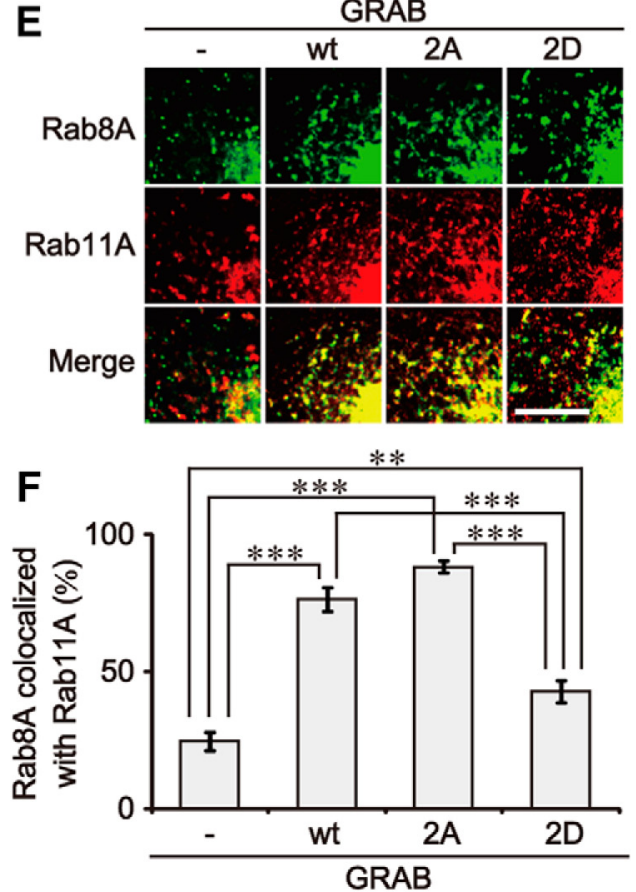

G

Time (s) $\quad \begin{array}{llllllll}0 & 4 & 8 & 12 & 16 & 20 & 24 & 28\end{array}$

\begin{tabular}{|c|c|c|c|c|c|c|c|c|}
\hline Rab8A & & $=$ & $\leftarrow$ & $\cdot k$ & $=$ & r. & 8 & 4 \\
\hline$a b 11 A$ & $x_{1}$ & $+t$ & 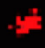 & * & 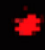 & 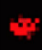 & $\Rightarrow$ & 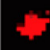 \\
\hline Merg & w & 4 & 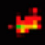 & $\Delta$ & $=$ & 학 & 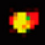 & 4 \\
\hline
\end{tabular}

H

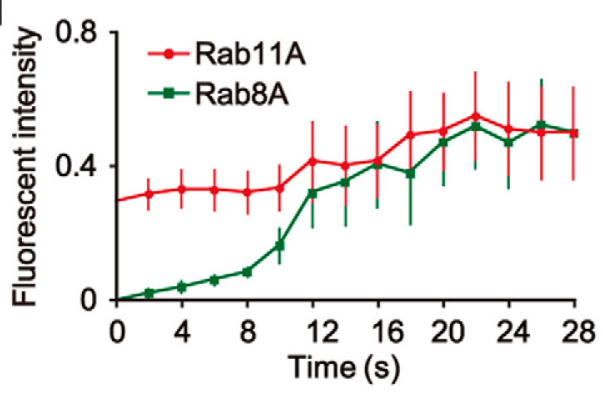

Figure 6. Effect of phosphorylation of GRAB on the recruitment of Rab8A- to Rab11A-positive endosomes. $A$, $C$, Effect of Cdk5-phosphorylatable sites in GRAB on its association with wt Rab8A ( $A$ ) or ci Rab8A (C). The extract (input) of cells expressing wt GRAB, GRAB 2A, or GRAB 2D (mStr-tagged) and EGFP-wt Rab8A (A) or EGFP-ci Rab8A (C). EGFP-Rab8A was immunoprecipitated with anti-GFP or control lgG (Cont), and GRAB and Rab8 were detected in the immunoprecipitate (IP) by immunoblotting with anti-GRAB and anti-GFP, respectively. $B, D$, Quantification of GRAB bound to wt Rab8A ( $\boldsymbol{B}$ ) or ci Rab8A (D). Data are mean \pm SEM. Each Rab8 isoform; $n=3$. $E$, Effect of phosphorylation of GRAB on the localization of Rab8A-into Rab11A-positive vesicles. EGFP-Rab8A and mStr-Rab11A were coexpressed with wt GRAB, GRAB 2A, or GRAB 2D. Control (-), no transfected GRAB. Scale bar, $10 \mu \mathrm{m}$. $\boldsymbol{F}$, Percentage of Rab8A localized in Rab11A-containing vesicles. Data are mean \pm SEM. Each experiment, $n=10$. G, Time course of EGFP-Rab8A accumulation on mStr-Rab11A-positive endosomes when coexpressed with wt GRAB in COS-7 cells. Scale bar, $1 \mu \mathrm{m}$. $\boldsymbol{H}$, Quantification with time of EGFP-Rab8A and mStr-Rab11A accumulation on mStr-Rab11A-positive endosomes when coexpressed with wt GRAB in COS-7 cells. Data are mean \pm SEM; $n=5$. ${ }^{*} p<0.05$. ${ }^{* *} p<0.01$. ${ }^{* * *} p<0.001$.

cles moved toward the growth cone; the vesicle indicated by the arrows moved in the distal direction along the axon until $8 \mathrm{~s}$ and then stopped, and the second vesicle, indicated by arrowheads was initially stationary and began to move at $8 \mathrm{~s}$ (Fig. 8A). On the other hand, whereas one vesicle labeled with only ca Rab11A moved bidirectionally, the other two ca Rab11A-labeled vesicles did not move (Fig. 8A). The observation indicates clearly that GRAB is transported on Rab11Apositive endosomes in the axon.
The above observation suggested a possibility that the association of GRAB alters the movement of Rab11-positive endosomes. Then, we analyzed the movement of GRAB-positive or negative and ca Rab11A-positive vesicles quantitatively. GRAB/ca Rab11A-double positive vesicles were $\sim 86 \%$ mobile, and GRAB-negative vesicles were $76 \%$ (Fig. $8 B$ ), suggesting that the GRAB-binding increases the ratio of moving vesicles. Among mobile vesicles, $27 \%$ of ca Rab11Alabeled vesicles moved anterogradely, $49 \%$ moved retrogradely, and $24 \%$ were bidirectional. The association of GRAB increased bidirec- 

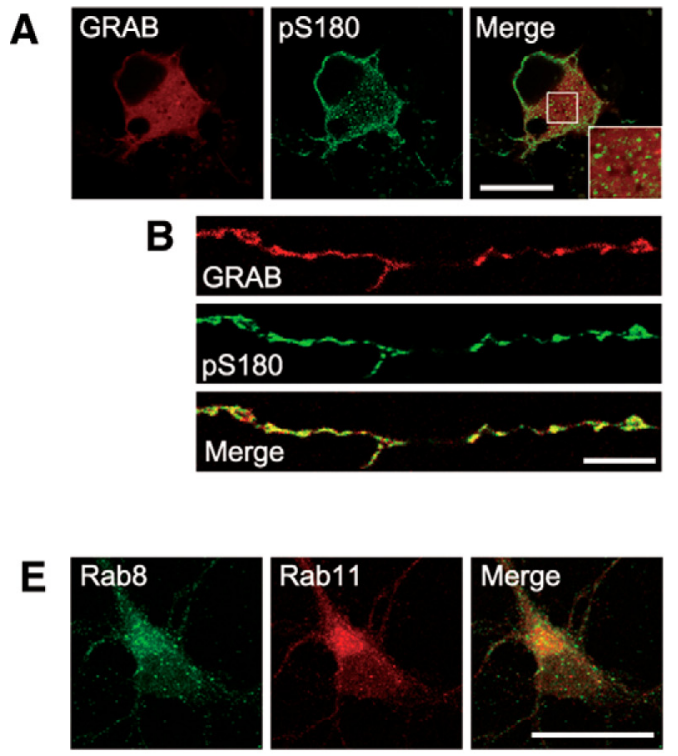
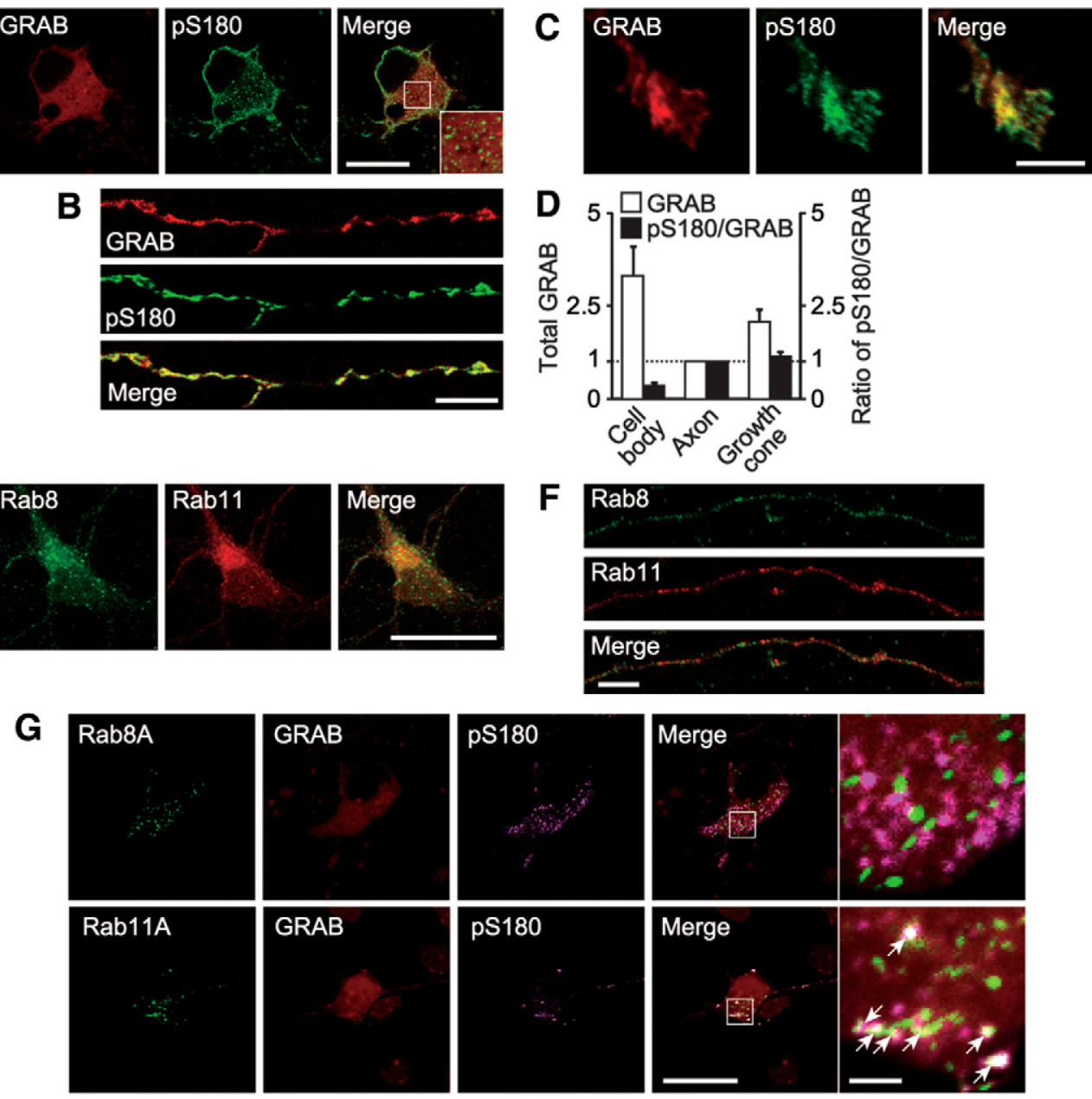

H
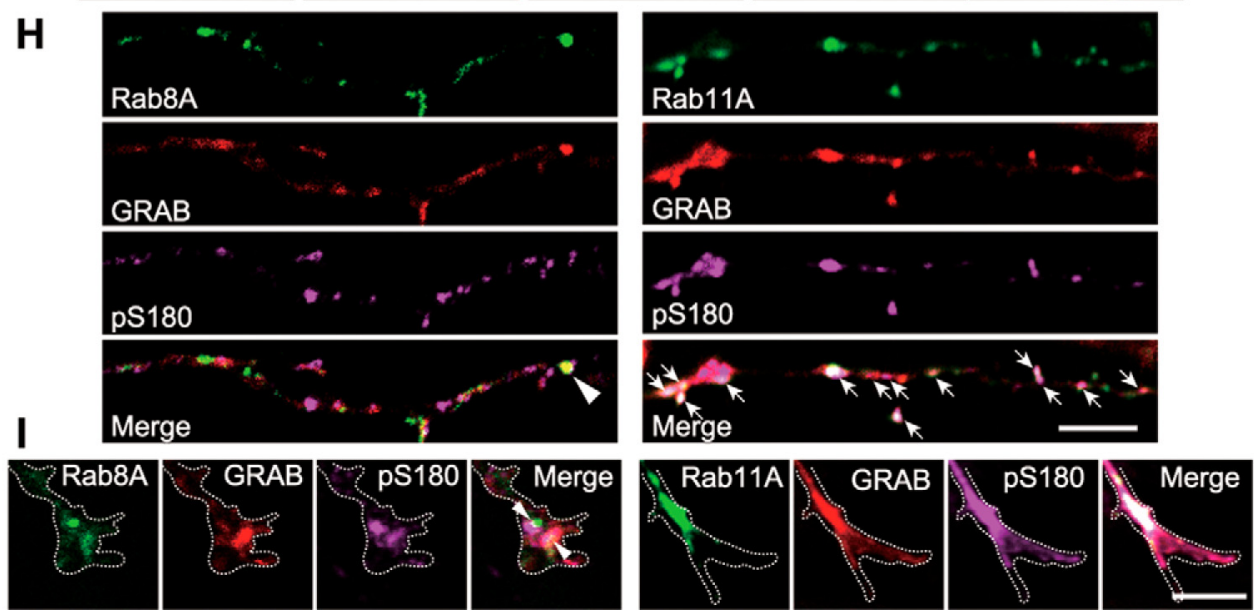

Figure 7. Subcellular locations of phosphorylated GRAB in neurons. $A-C$, Location of phosphorylated GRAB in neurons. Cortical neurons were transfected with $m S$ Str-GRAB at 2 DIV, then visualized by mStr fluorescence and immunostained with anti-pS180 at 3 DIV. A, Cell body. B, Axon. C, Growth cone. D, Total GRAB (white bar) and the ratio of phosphorylated GRAB to total GRAB (black bar) in the cell body, axon, and growth cone. Data are mean $\pm S E M ; n=20$ for each region. $E, F$, Immunolocalization of endogenous Rab8 and Rab11 in rat hippocampal neurons. $\boldsymbol{E}$, Cell body. $\boldsymbol{F}$, Axon. G-I, Colocalization of phosphorylated GRAB with Rab8A or Rab11A. mStr-GRAB was cotransfected with EGFP-Rab8A or EGFP-Rab11A into cortical neurons at 2 DIV and immunostained with anti-pS180 at 3 DIV. G, Cell body. $\boldsymbol{H}$, Axon. I, Growth cone. $\boldsymbol{H}$, Bottom right panels, Arrows indicate the colocalization of phosphorylated GRAB with Rab11A. Bottom left panel, Arrowhead indicates colocalized nonphosphorylated GRAB and Rab8A. I, Left merged panel, Arrowheads indicate vesicles of different intensities labeled with phosphorylated GRAB and Rab8A. Scale bars: $A, \boldsymbol{E}, \boldsymbol{G}$ (left merged panel), $20 \mu \mathrm{m} ; \boldsymbol{B}, \boldsymbol{C}, \boldsymbol{F}, \boldsymbol{H}, \boldsymbol{I}, 5 \mu \mathrm{m} ; \boldsymbol{G}$ (right merged panel), $2 \mu \mathrm{m}$.

tional movement significantly and anterograde movement slightly with the concomitant decrease in the retrograde movement (Fig. $8 C$ ). The binding of GRAB tended to decrease the velocity of the movement; the mean velocity was $0.75 \mu \mathrm{m} / \mathrm{s}$ for GRAB-positive and $0.84 \mu \mathrm{m} / \mathrm{s}$ for GRAB-negative vesicles (Fig. $8 D$ ). Thus, the associa- tion of GRAB modified the movement of ca Rab11A-positive vesicles. Considering that phosphorylated GRAB bound to Rab11A would be inactive for the GEF activity, GRAB may affect the movement of ca Rab11A-positive vesicles by a GEF activity-independent mechanism. 
A
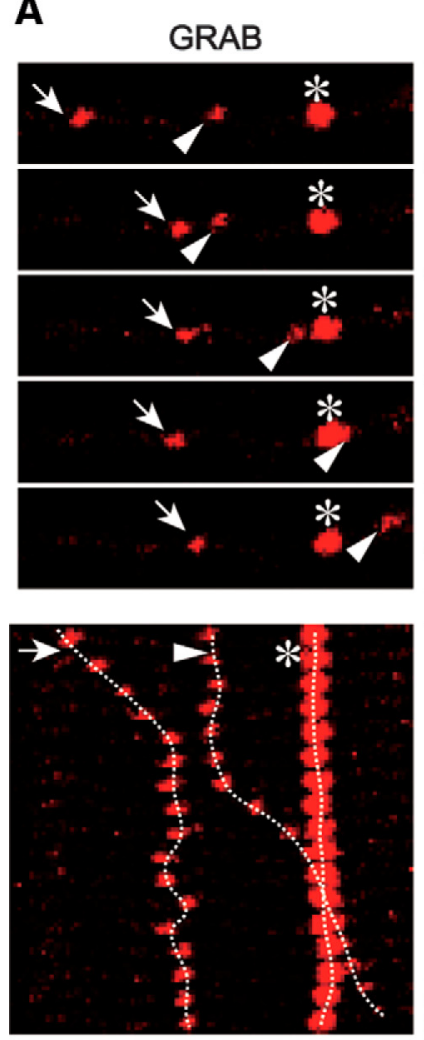

B

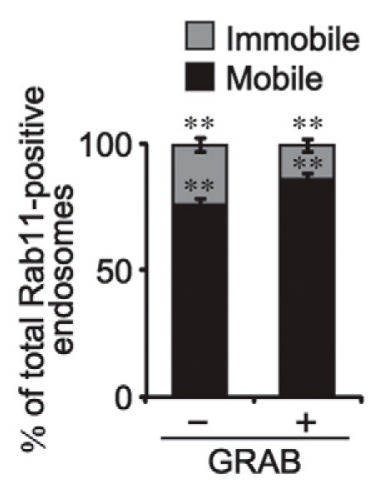

ca Rab11A
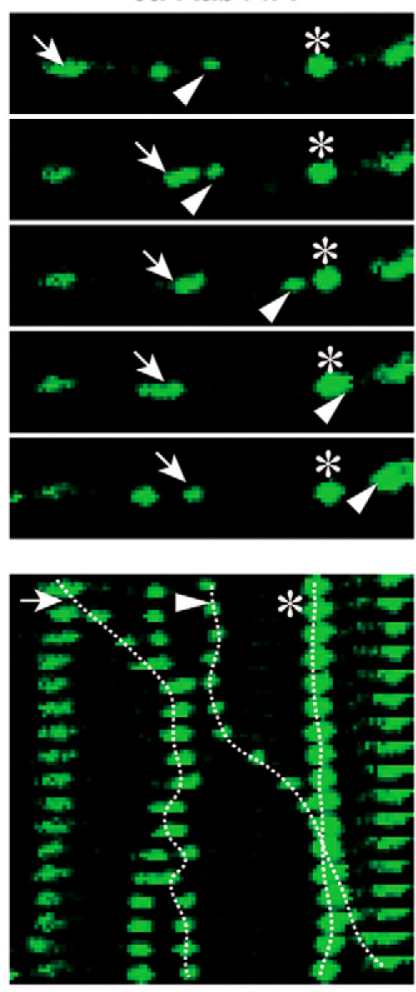

C

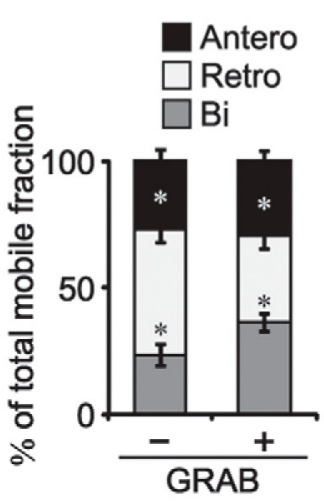

D
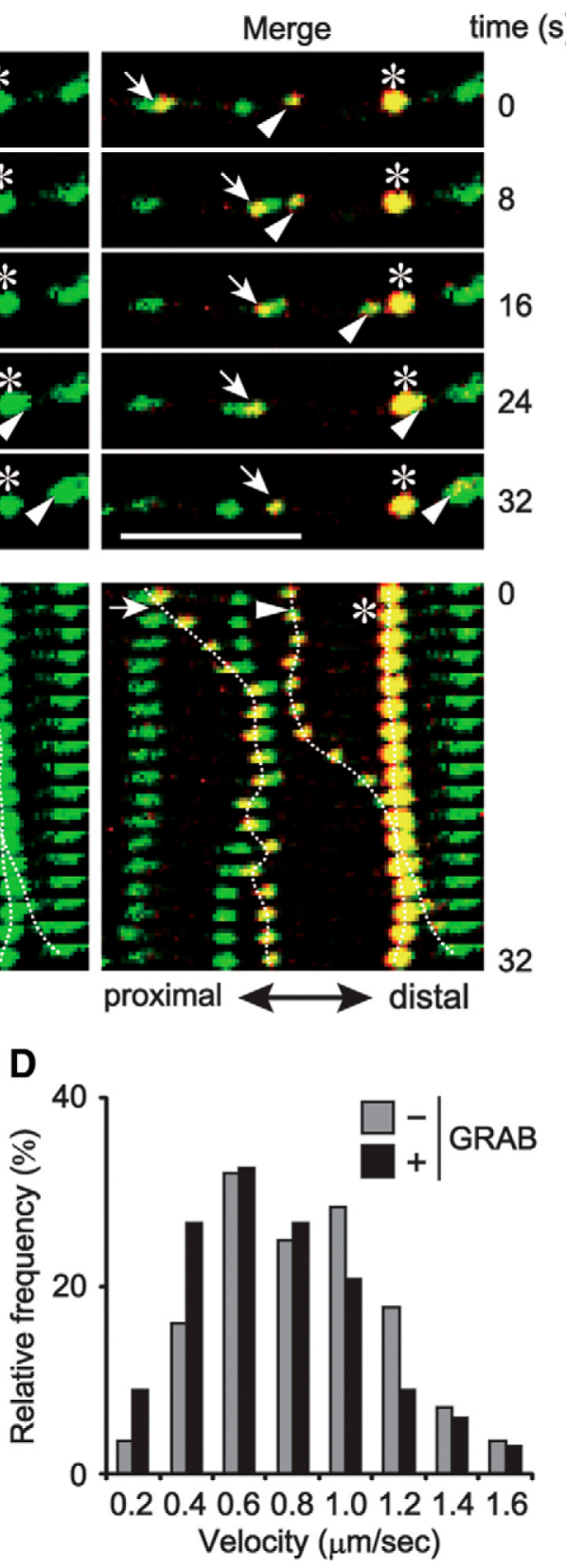

Figure 8. Cotransport of GRAB with ca Rab11A in an axon. A, mStr-GRAB was cotransfected with EGFP-ca Rab11A into cortical neurons at 2 DIV, and the transport of GRAB and ca Rab11A was observed in the axons at 4 DIV. ca Rab11A was visualized as EGFP fluorescence (middle panels) and GRAB as mStr fluorescence (left panels). Arrows, arrowheads, and asterisks indicate the three vesicles labeled by GRAB and Rab11A. Scale bar, $5 \mu \mathrm{m}$. Bottom panels, Kymographs for a 32 s observation period. The cell body is to the left. $B$, Percentage of moving or stationary Rab11A-positive vesicles in the axon. Data are mean \pm SEM. ca Rab11A-labeled vesicles, $n=205 ; \mathrm{GRAB} /$ ca Rab11-labeled vesicles, $n=200$. ${ }^{* *} p<0.01$. C, Percentage of Rab11A-positive endosomes moving anterogradely, retrogradely, or bidirectionally. ca Rab11A-labeled vesicles, $n=156 ; G R A B /$ ca Rab11-labeled vesicles, $n=171 .{ }^{*} p<0.05$. $D$, Velocity of Rab11A-positive endosomes in the axon. ca Rab11A-labeled vesicles, $n=75 ;$ GRAB/ca Rab11-labeled vesicles, $n=88$.

Cdk5-dependent GRAB phosphorylation regulates in vivo neuronal migration and process formation

To demonstrate in vivo function of GRAB and its phosphorylation, we used in utero electroporation approach for the study of neuronal migration. At first, we knocked GRAB down by electroporation of shGRAB $\# 1$, \#4, or SC into neural progenitors present in the ventricular zone at embryonic day 14 (E14), and the migration of differentiated neurons was scored at E17 (Fig. 9A-D). In control experiments with SC, $>90 \%$ of neurons exit the ventricular zone/subventricular zone and $58 \%$ of neurons reached the cortical plate $(\mathrm{CP})$. In contrast, in neurons electroporated with shGRAB \#1 or \#4, most neurons remained in lower re- gions of the cerebral cortex, such as the intermediate zone (IZ), without further migration into the CP (Fig. 9A,B). These results indicate that GRAB has a role in neuronal migration during brain development. Neurons in IZ displayed a multipolar morphology, and their movement was characterized by extending and retracting short processes (Tabata and Nakajima, 2003). We assessed the role of GRAB in the process formation of neurons in IZ. Whereas $>80 \%$ of control neurons display a short process oriented toward the marginal zone, such percentage was reduced to $\sim 60 \%$ when the knockdown vectors were introduced (Fig. 9C,D), indicating the involvement of GRAB in the process formation in vivo. 
A $\mathrm{E} 14-\mathrm{E} 17$
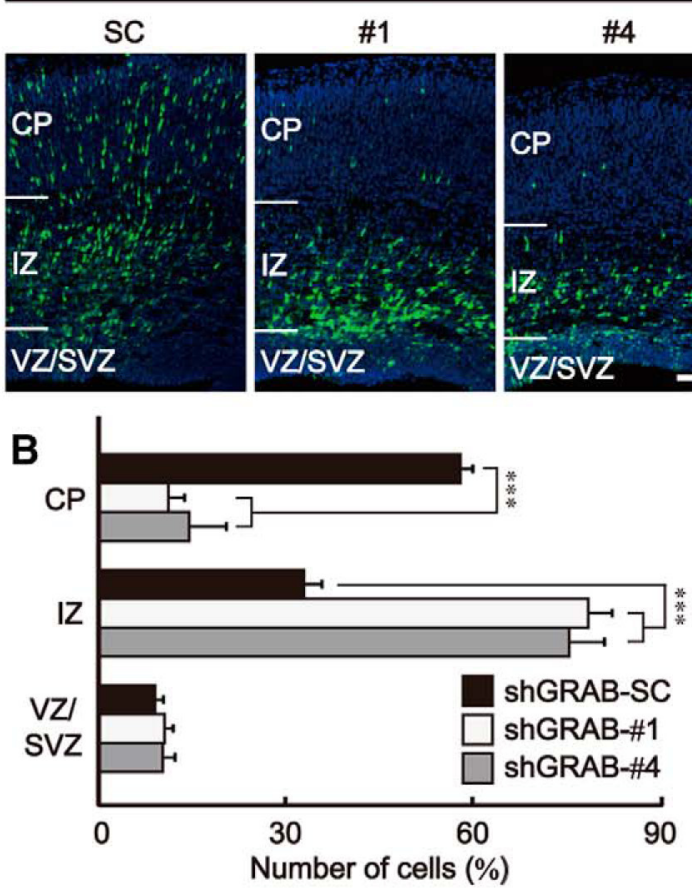

$\mathbf{E}$
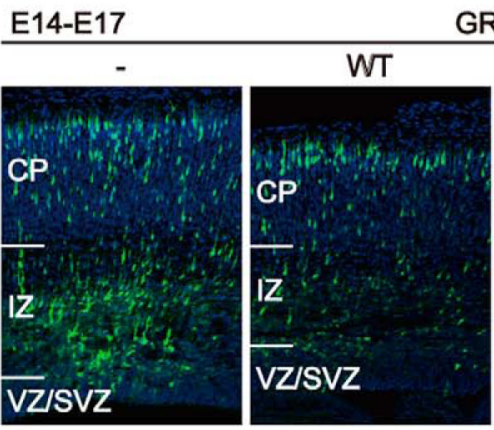

$\mathbf{F}$

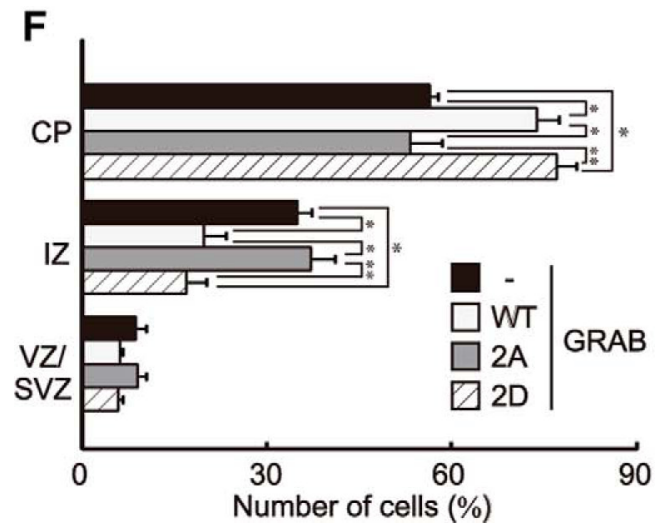

ShGRAB
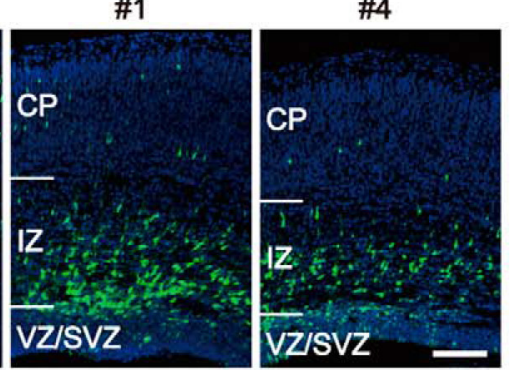

D
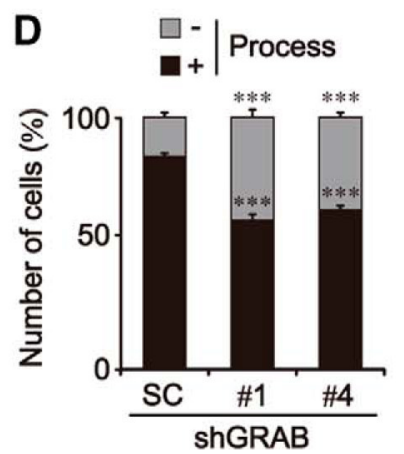

G

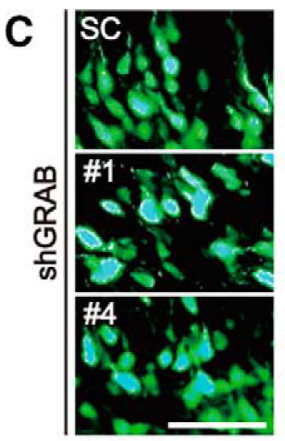

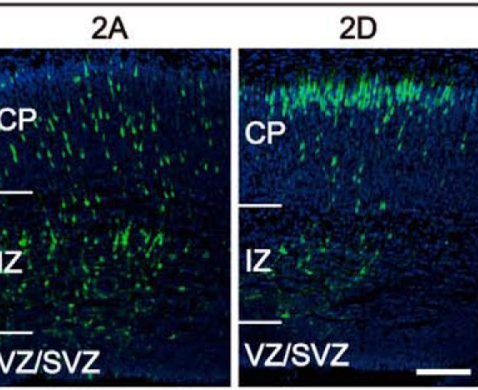

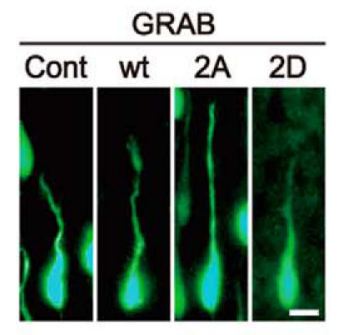

H

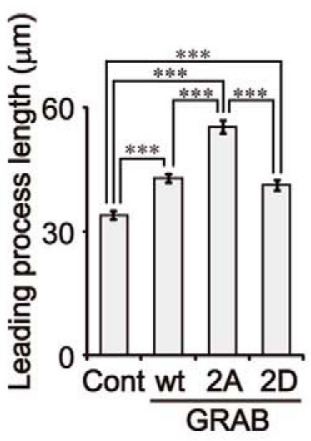

I

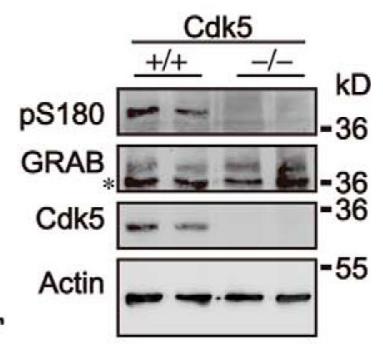

Figure 9. Role of GRAB and its phosphorylation in neuronal migration and process formation in vivo. $A$, Effect of GRAB knockdown on migration of cortical projection neurons. shGRAB \#1, \#4, or SC was coelectroporated into neural progenitor neurons in mouse embryonic brains at E14 with EGFP. Migration of neurons was observed at E17. The nucleus of neurons was stained with DAPI. CP, IZ, and ventricular zone/subventricular zone (VZ/SVZ) are indicated. Scale bar, $100 \mu \mathrm{m}$. B, Quantification of EGFP-expressing neurons in respective cortical layers. The percent ratio of total neurons was expressed as the mean $\pm S E M ; n>60$ neurons from four brains injected with respective shRNA. ${ }^{* *} p<0.001$. C, Representative images of neurons in $I Z$ of GRAB knocked-down mouse brain cortex. Scale bar, $50 \mu \mathrm{m}$. D, Quantification of neurons with or without processes. Data are mean $\pm S E M ; n>40$ neurons from four brains injected with respective shRNA. ${ }^{* * *} p<0.001$. $E$, Effect of phosphorylation of GRAB on neuronal migration. wt GRAB, GRAB 2A, or GRAB 2D was coelectroporated into brains of mouse at E14 with EGFP. Migration of neurons was observed at E17. The nucleus was stained with DAPI. Scale bar, $100 \mu \mathrm{m}$. $F$, Quantification of EGFP-expressing neurons in each cortical layer. The percent ratio was expressed as mean \pm SEM. Control or wt GRAB; $n>$ 40 neurons from four brains; GRAB 2A or 2D; $n>60$ neurons from five brains. ${ }^{*} p<0.05 .{ }^{* *} p<0.01$. G, Representative images of locomotive neurons in lower $(P$ at E17. Scale bar, $10 \mu \mathrm{m}$. $\boldsymbol{H}$, The lengths of a leading process. Data are mean \pm SEM. Each GRAB isoform; $n>100 .{ }^{* * *} p<0.001$. I, Phosphorylation of GRAB at Ser180 in brains of Cdk5-deficient mouse ( $-/-$ ). An asterisk in GRAB indicates nonspecific bands. Actin is the control. 
Next, we examined the role of phosphorylation of GRAB by expression of GRAB wt, $2 \mathrm{~A}$, or $2 \mathrm{D}$ in migrating neurons (Fig. $9 E-H)$. GRAB $2 \mathrm{D}$ as well as wt increased the number of neurons that reach the $\mathrm{CP}$. In contrast, GRAB 2A did not affect the migration; the number of neurons counted in three regions was similar to those of control mice expressing EGFP (Fig. $9 E, F$ ). The results indicate that the phosphorylation regulates the GRAB activity in neuronal migration. We measured the length of a leading process of locomotive neurons in CP. Typical examples of them are shown in Figure 9G. Expression of GRAB wt or GRAB 2D increased the length of the leading process slightly compared with control neurons, and GRAB 2A increased the length more significantly, $\sim 1.6$-fold of control neurons (Fig. 9H). Finally, we confirmed the in vivo phosphorylation of GRAB by Cdk5 using $C d k 5^{-l-}$ mouse brains. Anti-pS180 reactive GRAB in wild-type mouse was greatly reduced in $\mathrm{Cdk5^{-- }}$ mouse brain (Fig. 9I). Thus, GRAB is a substrate for Cdk5 in mouse brains. These results indicate that GRAB plays a role in the migration of cerebral cortical neurons likely through the process formation and phosphorylation by Cdk 5 regulates the GRAB activity.

\section{Discussion}

Here we have shown that the GRAB-mediated Rab11A-Rab8A interaction constitutes a novel Rab cascade regulating axonal outgrowth and that Cdk5 is an upstream regulator of the pathway. Rab8 is involved in the secretory exocytic pathway from the trans Golgi network to the surface membrane (Ang et al., 2003; Sato et al., 2007). Rab8 has been reported to promote neurite elongation (Huber et al., 1995), but its function(s) has not been further assessed. Herein, we confirmed those results by showing that Rab8A promoted axonal outgrowth in cortical neurons. Conversely, Rab11 functions as a conventional Rab that regulates the transport of recycling endosomes from sorting endosomes to surface membranes via the perinuclear endosomal compartment in cultured cells (Li and DiFiglia, 2012). Rab11 participation in axon elongation was recently reported (Ascano et al., 2009; Eva et al., 2010; Takano et al., 2012, 2014). We observed that Rab11Alabeled vesicles bud from the perinuclear endocytic region located at the axon base (Takano et al., 2014). Because the Golgi apparatus is also found there, both endocytic and exocytic vesicles could travel from the perinuclear region of neurons to their axon tips. In contrast to cultured nonpolarized cells, both endocytic and secretory vesicles in axons are conveyed along a limited number of microtubules. Thus, it was intriguing to know whether the two types of vesicles are transported independently or together. Our observations indicate that Rab11-positive vesicles are transported separately from Rab8-positive vesicles in axons. Although it is not clear how Rab8 is transported, it appears to be inactive during its transport in axon and activated at the axon terminus.

We showed that GRAB stimulates axons outgrowth by mediating the interaction between Rab11 and Rab8. GRAB is known to be a Rab8 GEF and a Rab11-binding partner (Yoshimura et al., 2010; Guo et al., 2013; Horgan et al., 2013). However, the GRAB Rab8-GEF and Rab11-binding activities were independently examined in the aforementioned reports, leaving open the question of whether these activities are linked. Further, GRAB had not been examined for the role in neurite formation. Our study is the first to determine a biological relationship between GRAB and its related Rabs, even though GRAB had previously been proposed to regulate synaptic vesicle movement in the presynaptic region (Luo et al., 2001). Moreover, we have shown that Cdk5 regulates GRAB GEF activity, which is the first demonstration of phosphorylation-dependent regulation of GRAB GEF activity. Cdk5-p35 is a protein kinase preferentially expressed in neurons, and it regulates neurite outgrowth via cytoskeletal reorganization partly by targeting a number of GEFs for Racl (Nikolic et al., 1998; Rashid et al., 2001; Mokalled et al., 2010; Fang et al., 2011; Tang et al., 2014). In contrast, recent studies indicated that Cdk5p35 also regulates membrane transport in axon and dendrites (Ou et al., 2010; Goodwin et al., 2012; Klinman and Holzbaur, 2015). That Cdk5-p35 is membrane bound and accumulates at the perinuclear membrane region (Patrick et al., 1999; Asada et al., 2008) supports its role in membrane trafficking. Its association with the Golgi apparatus may also suggest participation in exocytic membrane delivery (Paglini et al., 2001). In particular, its close relationship to Rab11 function seemed obvious. We showed that lemur kinase 1A (LMTK1A), a Cdk5-p35 substrate, suppresses transport of Rab11-positive vesicles in axons and dendrites in a Cdk5-dependent manner (Takano et al., 2012, 2014). Herein, we showed that Cdk5-p35 regulates the Rab11-effector GRAB by phosphorylation. Thus, Cdk 5 modulates the transport of Rab11-dependent endosomes upstream and downstream, suggesting that Cdk5 activity mediates Rab11-dependent vesicle transport.

Nonphosphorylated GRAB, which can bind active Rab11A, activates Rab8A and recruits it to Rab11A-positive vesicles. This handoff seems to occur in the growth cone, allowing vesicles driven by Rab11A to be passed off to Rab8A for fusion with plasma membranes (Fig. 10). This pass-forward interaction has been shown for Rab5-Rab7 endocytic trafficking in the lysosomal pathway (Hutagalung and Novick, 2011). Although a Rab11ARab8A cascade has not been found for canonical endosomal recycling or exocytic pathways, its occurrence was suggested for AMPA-receptor transport involving Rab11-dependent transport in the dendritic shaft to Rab8-dependent insertion into the postsynaptic membrane in spines (Brown et al., 2007). The Rab11-Rab8 cascade also operates during ciliogenesis. The ciliary Rab11-Rab8 cascade is regulated by Rabin8 GEF-Rab8 activity (Knödler et al., 2010; Westlake et al., 2011; Wang et al., 2012; Chiba et al., 2013). Very recently, it is shown that Rabin8 also regulates neurite outgrowth by coordinating Rab8 with Rab11 in PC12 cells (Homma and Fukuda, 2016). The sequence of Rabin8 is $46 \%$ identical to that of GRAB, and it also can bind Rab11. However, its activation mechanism is different from that of GRAB as discussed below.

GRAB GEF activity is suppressed by Cdk5-p35 phosphorylation. In contrast, ERK1/2 phosphorylation of Rabin8 activates it (Wang et al., 2015). ERK1/2 is also a proline-directed kinase with overlapping phosphorylation consensus sequences with Cdk5. ERK1/2 phosphorylates Rabin8 at four sites: Ser 16 and Ser 19 near the N terminus, and Ser247 and Ser250 C-terminal to the Rabin8 GEF domain. The two N-terminal phosphorylation sequences are not present in GRAB, whereas the other two are conserved in GRAB, although the sequences are not identical. The Ser250 ERK-phosphorylation site in Rabin8 corresponds to the Ser169 Cdk5-phosphorylation site in GRAB. Given the opposing effects of phosphorylation that these two kinases have on GEF activities suggests that the $\mathrm{N}$-terminal phosphorylation sites in Rabin8 may be involved in its activation. Regulation of their kinase activities also differs for ERK1/2 and Cdk5-p35. ERK1/2 is activated transiently by growth factors, whereas Cdk 5 is constitutively active in the form of Cdk5-p35 (Hisanaga and Endo, 2010). Ciliogenesis, involving ERK1/2-Rabin8, is a cell cycle-dependent event, which is promoted by growth factors. Therefore, "activation" of Rabin8 is a dynamic regulatory mechanism controlled by ERK1/2. In 


\section{Phosphorylation of GRAB recruitment of Rab8}

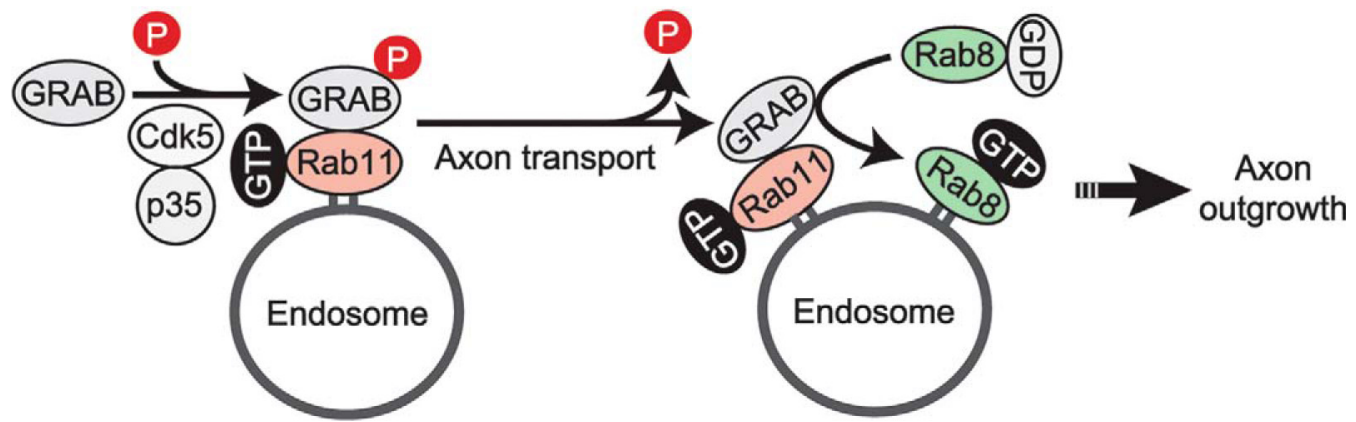

Figure 10. Schematic of the forward handoff mechanism by GRAB in endosomal vesicles at the axonal tip. Cdk5-p35 increases the association of GRAB with Rab11A by phosphorylating GRAB. Phosphorylated GRAB is transported with Rab11A on vesicles through the axon from the cell body to its growth cone where the GEF activity of GRAB is activated by dephosphorylation so as to recruit Rab8A to Rab11A-positive endosomes.

contrast, GRAB needs to be inactive while it is transported within long neuronal axons to avoid inappropriate activation of Rab8. To fulfill this requirement, a constitutive active and membranebound kinase (i.e., Cdk5-p35) would be used.

We have shown here that GRAB regulates the neuronal migration in embryonic mouse brains. The results have several, at least triple, meanings: (1) in vivo function of GRAB, (2) the role of the exocytic membrane transport in neuronal migration, and (3) Cdk5-dependent regulation of neuronal migration via membrane trafficking. (1) This is the first evidence indicating physiological function of GRAB in brains. In particular, the effects of GRAB expression on short process formation in IZ neurons and of the phosphorylation on the length of the leading process in locomotive neurons substantiate its in vivo role in axon outgrowth. GRAB may play a role in the formation of various types of processes through supplying membrane components to growing tip. (2) The involvement of endocytic Rabs, Rab5, Rab7, and Rab11 in neuronal migration is already shown (Kawauchi et al., 2010). However, there is no report on exocytic Rabs. GRAB is a GEF of exocytic Rab8. Together, the data indicate that endocytic and exocytic cycles of membrane component are necessary for neuronal migration. (3) Layer formation of cortical neurons is disrupted in Cdk5 $5^{-1-}$ mouse brains (Ohshima et al., 1996; Gilmore et al., 1998). Several Cdk5 substrates, such as FAK, doublecortin, p2 $7^{\mathrm{kip} 1}$, drebrin, and Mst3, have been reported as possible downstream pathways (Xie et al., 2003; Tanaka et al., 2004; Kawauchi et al., 2006; Tanabe et al., 2014; Tang et al., 2014). Considering that $\mathrm{Cdk} 5$ is a membrane-bound kinase, however, it is also likely that Cdk5 regulates neuronal migration through membrane proteins and/or membrane trafficking. GRAB is a Cdk5 substrate, which regulates membrane transport.

Axon outgrowth accompanies surface expansion, which takes place by addition of new membrane components at the tips of neurites. The membranes to be inserted are transported as small vesicles in axons from the cell bodies. Even though axons appear to be narrow tubes, their trafficking system is complicated. Their membrane supplies are derived from endocytic and secretory vesicles, and many Rabs (e.g., Rab6, Rab8, Rab10, Rab11, Rab27, Rab33, and Rab35) are involved (Huber et al., 1995; Arimura et al., 2009; Schlager et al., 2010; Wang et al., 2011; Kobayashi and Fukuda, 2012; Nakazawa et al., 2012; Takano et al., 2012). Rab35 supports Cdc42 activation, an essential feature during axon specification and elongation (Villarroel-Campos et al., 2016b). Because most studies concerning membrane transport have focused on one or a few Rabs, little is known concerning the interactions among Rabs and their regulatory mechanisms, although a recent study showed that several Rabs form clusters in the neurites of NGF-treated PC12 cells (Kobayashi et al., 2014). In these clusters, Rab35 recruits Rab8, Rab13, and Rab36 to Arf6-positive recycling endosomes in conjunction with an MICAL-L1 scaffold. We have now extended the knowledge of Rab interactions by showing the ordered interaction between Rab11 and Rab8 in the axons of developing neurons. Other Rab-Rab interactions would also be used for proper delivery of the distinct membrane components during axon outgrowth.

\section{References}

Ang AL, Fölsch H, Koivisto UM, Pypaert M, Mellman I (2003) The Rab8 GTPase selectively regulates AP-1B-dependent basolateral transport in polarized Madin-Darby canine kidney cells. J Cell Biol 163:339-350. CrossRef Medline

Arimura N, Kimura T, Nakamuta S, Taya S, Funahashi Y, Hattori A, Shimada A, Ménager C, Kawabata S, Fujii K, Iwamatsu A, Segal RA, Fukuda M, Kaibuchi K (2009) Anterograde transport of TrkB in axons is mediated by direct interaction with Slp1 and Rab27. Dev Cell 16:675-686. CrossRef Medline

Asada A, Yamamoto N, Gohda M, Saito T, Hayashi N, Hisanaga S (2008) Myristoylation of p39 and p35 is a determinant of cytoplasmic or nuclear localization of active cyclin-dependent kinase 5 complexes. J Neurochem 106:1325-1336. CrossRef Medline

Ascaño M, Richmond A, Borden P, Kuruvilla R (2009) Axonal targeting of Trk receptors via transcytosis regulates sensitivity to neurotrophin responses. J Neurosci 29:11674-11685. CrossRef Medline

Bakos J, Bacova Z, Grant SG, Castejon AM, Ostatnikova D (2015) Are molecules involved in neuritogenesis and axon guidance related to autism pathogenesis? Neuromolecular Med 17:297-304. CrossRef Medline

Bellon A (2007) New genes associated with schizophrenia in neurite formation: a review of cell culture experiments. Mol Psychiatry 12:620-629. CrossRef Medline

Blümer J, Rey J, Dehmelt L, Mazel T, Wu YW, Bastiaens P, Goody RS, Itzen A (2013) RabGEFs are a major determinant for specific Rab membrane targeting. J Cell Biol 200:287-300. CrossRef Medline

Brown TC, Correia SS, Petrok CN, Esteban JA (2007) Functional compartmentalization of endosomal trafficking for the synaptic delivery of AMPA receptors during long-term potentiation. J Neurosci 27:13311-13315. CrossRef Medline

Chiba S, Amagai Y, Homma Y, Fukuda M, Mizuno K (2013) NDR2mediated Rabin8 phosphorylation is crucial for ciliogenesis by switching binding specificity from phosphatidylserine to Sec15. EMBO J 32:874885. CrossRef Medline

Conde C, Cáceres A (2009) Microtubule assembly, organization and dynamics in axons and dendrites. Nat Rev Neurosci 10:319-332. CrossRef Medline 
Contreras-Vallejos E, Utreras E, Bórquez DA, Prochazkova M, Terse A, Jaffe H, Toledo A, Arruti C, Pant HC, Kulkarni AB, González-Billault C (2014) Searching for novel Cdk5 substrates in brain by comparative phosphoproteomics of wild type and Cdk5 $5^{-1-}$ mice. PLoS One 9:e90363. CrossRef Medline

Dotti CG, Sullivan CA, Banker GA (1988) The establishment of polarity by hippocampal neurons in culture. J Neurosci 8:1454-1468. Medline

Eva R, Dassie E, Caswell PT, Dick G, ffrench-Constant C, Norman JC, Fawcett JW (2010) Rab11 and its effector Rab coupling protein contribute to the trafficking of beta 1 integrins during axon growth in adult dorsal root ganglion neurons and PC12 cells. J Neurosci 30:11654-11669. CrossRef Medline

Fang WQ, Ip JP, Li R, Ng YP, Lin SC, Chen Y, Fu AK, Ip NY (2011) Cdk5mediated phosphorylation of Axin directs axon formation during cerebral cortex development. J Neurosci 31:13613-13624. CrossRef Medline

Fukuda M (2003) Distinct Rab binding specificity of Rim1, Rim2, rabphilin, and Noc2: identification of a critical determinant of Rab3A/Rab27A recognition by Rim2. J Biol Chem 278:15373-15380. CrossRef Medline

Fukuda M, Mikoshiba K (1999) A novel alternatively spliced variant of synaptotagmin VI lacking a transmembrane domain. Implications for distinct functions of the two isoforms. J Biol Chem 274:31428-31434. CrossRef Medline

Fukuda M, Kanno E, Ishibashi K, Itoh T (2008) Large scale screening for novel rab effectors reveals unexpected broad Rab binding specificity. Mol Cell Proteomics 7:1031-1042. CrossRef Medline

Furusawa K, Asada A, Saito T, Hisanaga S (2014) The effect of Cyclindependent kinase 5 on voltage-dependent calcium channels in PC12 cells varies according to channel type and cell differentiation state. J Neurochem 130:498-506. CrossRef Medline

Gilmore EC, Ohshima T, Goffinet AM, Kulkarni AB, Herrup K (1998) Cyclin-dependent kinase 5-deficient mice demonstrate novel developmental arrest in cerebral cortex. J Neurosci 18:6370-6377. Medline

Goodwin PR, Sasaki JM, Juo P (2012) Cyclin-dependent kinase 5 regulates the polarized trafficking of neuropeptide-containing dense-core vesicles in Caenorhabditis elegans motor neurons. J Neurosci 32:8158-8172. CrossRef Medline

Guo Z, Hou X, Goody RS, Itzen A (2013) Intermediates in the guanine nucleotide exchange reaction of Rab8 protein catalyzed by guanine nucleotide exchange factors Rabin8 and GRAB. J Biol Chem 288:3246632474. CrossRef Medline

Hisanaga S, Endo R (2010) Regulation and role of cyclin-dependent kinase activity in neuronal survival and death. J Neurochem 115:1309-1321. CrossRef Medline

Homma Y, Fukuda M (2016) Rabin8 regulates neurite outgrowth in both a GEF-activity-dependent and -independent manner. Mol Biol Cell 27: 2107-2118.

Horgan CP, Hanscom SR, McCaffrey MW (2013) GRAB is a binding partner for the Rab1la and Rab11b GTPases. Biochem Biophys Res Commun 411:214-219. CrossRef Medline

Horton AC, Ehlers MD (2003) Neuronal polarity and trafficking. Neuron 40:277-295. CrossRef Medline

Hosokawa T, Saito T, Asada A, Fukunaga K, Hisanaga S (2010) Quantitative measurement of in vivo phosphorylation states of Cdk 5 activator $\mathrm{p} 35$ by Phos-tag SDS-PAGE. Mol Cell Proteomics 9:1133-1143. CrossRef Medline

Huber LA, Dupree P, Dotti CG (1995) A deficiency of the small GTPase rab8 inhibits membrane traffic in developing neurons. Mol Cell Biol 15:918924. CrossRef Medline

Hutagalung AH, Novick PJ (2011) Role of Rab GTPases in membrane traffic and cell physiology. Physiol Rev 91:119-149. CrossRef Medline

Kaminosono S, Saito T, Oyama F, Ohshima T, Asada A, Nagai Y, Nukina N, Hisanaga S (2008) Suppression of mutant Huntingtin aggregate formation by $\mathrm{Cdk} 5 / \mathrm{p} 35$ through the effect on microtubule stability. J Neurosci 28:8745-8755. CrossRef Medline

Kawauchi T (2014) Cdk5 regulates multiple cellular events in neural development, function and disease. Dev Growth Differ 56:335-348. CrossRef Medline

Kawauchi T, Chihama K, Nabeshima Y, Hoshino M (2006) Cdk5 phosphorylates and stabilizes p27kip 1 contributing to actin organization and cortical neuronal migration Nat Cell Biol 8:17-26. CrossRef Medline

Kawauchi T, Sekine K, Shikanai M, Chihama K, Tomita K, Kubo K, Nakajima K, Nabeshima Y, Hoshino M (2010) Rab GTPases-dependent endocytic pathways regulate neuronal migration and maturation through $\mathrm{N}$-cadherin trafficking. Neuron 67:588-602. CrossRef Medline

Kinoshita E, Kinoshita-Kikuta E, Takiyama K, Koike T (2006) Phosphatebinding tag, a new tool to visualize phosphorylated proteins. Mol Cell Proteomics 5:749-757. CrossRef Medline

Klinman E, Holzbaur EL (2015) Stress-induced CDK5 activation disrupts axonal transport via Lis1/Ndel1/Dynein. Cell Rep 12:462-473. CrossRef Medline

Klöpper TH, Kienle N, Fasshauer D, Munro S (2012) Untangling the evolution of Rab $G$ proteins: implications of a comprehensive genomic analysis. BMC Biol 10:71. CrossRef Medline

Knödler A, Feng S, Zhang J, Zhang X, Das A, Peränen J, Guo W (2010) Coordination of Rab8 and Rab11 in primary ciliogenesis. Proc Natl Acad Sci U S A 107:6346-6351. CrossRef Medline

Kobayashi H, Fukuda M (2012) Rab35 regulates Arf6 activity through centaurin- $\beta 2$ (ACAP2) during neurite outgrowth. J Cell Sci 125:22352243. CrossRef Medline

Kobayashi H, Etoh K, Ohbayashi N, Fukuda M (2014) Rab35 promotes the recruitment of Rab8, Rab13 and Rab36 to recycling endosomes through MICAL-L1 during neurite outgrowth. Biol Open 3:803-814. CrossRef Medline

Lewis TL Jr, Courchet J, Polleux F (2013) Cell biology in neuroscience: cellular and molecular mechanisms underlying axon formation, growth, and branching. J Cell Biol 202:837-848. CrossRef Medline

Li X, DiFiglia M (2012) The recycling endosome and its role in neurological disorders. Prog Neurobiol 97:127-141. CrossRef Medline

Luo HR, Saiardi A, Nagata E, Ye K, Yu H, Jung TS, Luo X, Jain S, Sawa A, Snyder SH (2001) GRAB: a physiologic guanine nucleotide exchange factor for Rab3A, which interacts with inositol hexakisphosphate kinase. Neuron 31:439-451. CrossRef Medline

Mokalled MH, Johnson A, Kim Y, Oh J, Olson EN (2010) Myocardinrelated transcription factors regulate the $\mathrm{Cdk} 5 /$ Pctaire 1 kinase cascade to control neurite outgrowth, neuronal migration and brain development. Development 137:2365-2374. CrossRef Medline

Mori Y, Matsui T, Furutani Y, Yoshihara Y, Fukuda M (2012) Small GTPase Rab17 regulates dendritic morphogenesis and postsynaptic development of hippocampal neurons. J Biol Chem 287:8963-8973. CrossRef Medline

Mori Y, Matsui T, Fukuda M (2013) Rabex-5 protein regulates dendritic localization of small GTPase Rab17 and neurite morphogenesis in hippocampal neurons. J Biol Chem 288:9835-9847. CrossRef Medline

Nakazawa H, Sada T, Toriyama M, Tago K, Sugiura T, Fukuda M, Inagaki N (2012) Rab33a mediates anterograde vesicular transport for membrane exocytosis and axon outgrowth. J Neurosci 32:12712-12725. CrossRef Medline

Nikolic M, Chou MM, Lu W, Mayer BJ, Tsai LH (1998) The p35/Cdk5 kinase is a neuron-specific Rac effector that inhibits Pak1 activity. Nature 395:194-198. CrossRef Medline

Ohshima T, Ward JM, Huh CG, Longenecker G, Veeranna, Pant HC, Brady RO, Martin LJ, Kulkarni AB (1996) Targeted disruption of the cyclindependent kinase 5 gene results in abnormal corticogenesis, neuronal pathology and perinatal death. Proc Natl Acad Sci U S A 93:11173-11178. CrossRef Medline

Ou CY, Poon VY, Maeder CI, Watanabe S, Lehrman EK, Fu AK, Park M, Fu WY, Jorgensen EM, Ip NY, Shen K (2010) Two cyclin-dependent kinase pathways are essential for polarized trafficking of presynaptic components. Cell 141:846-858. CrossRef Medline

Paglini G, Peris L, Diez-Guerra J, Quiroga S, Cáceres A (2001) The Cdk5p35 kinase associates with the Golgi apparatus and regulates membrane traffic. EMBO Rep 2:1139-1144. CrossRef Medline

Patrick GN, Zukerberg L, Nikolic M, de la Monte S, Dikkes P, Tsai LH (1999) Conversion of p 35 to $\mathrm{p} 25$ deregulates Cdk5 activity and promotes neurodegeneration. Nature 402:615-622. CrossRef Medline

Pfenninger KH (2009) Plasma membrane expansion: a neuron's Herculean task. Nat Rev Neurosci 10:251-261. CrossRef Medline

Rashid T, Banerjee M, Nikolic M (2001) Phosphorylation of Pak1 by the p35/Cdk5 kinase affects neuronal morphology. J Biol Chem 276:4904349052. CrossRef Medline

Sann S, Wang Z, Brown H, Jin Y (2009) Roles of endosomal trafficking in neurite outgrowth and guidance. Trends Cell Biol 19:317-324. CrossRef Medline

Sano H, Roach WG, Peck GR, Fukuda M, Lienhard GE (2008) Rab10 in 
insulin-stimulated GLUT4 translocation. Biochem J 411:89-95. CrossRef Medline

Sato T, Mushiake S, Kato Y, Sato K, Sato M, Takeda N, Ozono K, Miki K, Kubo Y, Tsuji A, Harada R, Harada A (2007) The Rab8 GTPase regulates apical protein localization in intestinal cells. Nature 448:366-369. CrossRef Medline

Schlager MA, Kapitein LC, Grigoriev I, Burzynski GM, Wulf PS, Keijzer N, de Graaff E, Fukuda M, Shepherd IT, Akhmanova A, Hoogenraad CC (2010) Pericentrosomal targeting of Rab6 secretory vesicles by BicaudalD-related protein 1 (BICDR-1) regulates neuritogenesis. EMBO J 29: 1637-1651. CrossRef Medline

Smith DS, Tsai LH (2002) Cdk5 behind the wheel: a role in trafficking and transport? Trends Cell Biol 12:28-36. CrossRef Medline

Stenmark H (2009) Rab GTPases as coordinators of vesicle traffic. Nat Rev Mol Cell Biol 10:513-525. CrossRef Medline

Tabata H, Nakajima K (2003) Multipolar migration: the third mode of radial neuronal migration in the developing cerebral cortex. J Neurosci 23:9996-10001. Medline

Takai Y, Sasaki T, Matozaki T (2001) Small GTP-binding proteins. Physiol Rev 81:153-208. Medline

Takano T, Tomomura M, Yoshioka N, Tsutsumi K, Terasawa Y, Saito T, Kawano H, Kamiguchi H, Fukuda M, Hisanaga S (2012) LMTK1/ AATYK1 is a novel regulator of axonal outgrowth that acts via Rab11 in a Cdk5-dependent manner. J Neurosci 32:6587-6599. CrossRef Medline

Takano T, Urushibara T, Yoshioka N, Saito T, Fukuda M, Tomomura M, Hisanaga S (2014) LMTK1 regulates dendritic formation by regulating movement of Rab11A-positive endosomes. Mol Biol Cell 25:1755-1768. CrossRef Medline

Tanabe K, Yamazaki H, Inaguma Y, Asada A, Kimura T, Takahashi J, Taoka M, Ohshima T, Furuichi T, Isobe T, Nagata K, Shirao T, Hisanaga S (2014) Phosphorylation of drebrin by cyclin-dependent kinase 5 and its role in neuronal migration. PLoS One 9:e92291. CrossRef Medline

Tanaka T, Serneo FF, Tseng HC, Kulkarni AB, Tsai LH, Gleeson JG (2004) Cdk5 phosphorylation of doublecortin ser297 regulates its effect on neuronal migration Neuron 41:215-227. CrossRef

Tang J, Ip JP, Ye T, Ng YP, Yung WH, Wu Z, Fang W, Fu AK, Ip NY (2014) Cdk5-dependent Mst3 phosphorylation and activity regulate neuronal migration through RhoA inhibition. J Neurosci 34:7425-7436. CrossRef Medline

Villarroel-Campos D, Gastaldi L, Conde C, Cáceres A, Gonzalez-Billault C
(2014) Rab-mediated trafficking role in neurite formation. J Neurochem 120:240-248. CrossRef Medline

Villarroel-Campos D, Bronfman FC, Gonzalez-Billault C (2016a) Rab GTPase signaling in neurite outgrowth and axon specification. Cytoskeleton 73:498-507. CrossRef Medline

Villarroel-Campos D, Henríquez DR, Bodaleo FJ, Oguchi ME, Bronfman FC, Fukuda M, Gonzalez-Billault C (2016b) Rab35 functions in axon elongation are regulated by P53-related protein kinase in a mechanism that involves Rab35 protein degradation and the microtubule-associated protein 1B. J Neurosci 36:7298-7313. CrossRef Medline

Wang J, Morita Y, Mazelova J, Deretic D (2012) The Arf GAP ASAP1 provides a platform to regulate Arf4- and Rab11-Rab8-mediated ciliary receptor targeting. EMBO J 75:5-10. CrossRef Medline

Wang J, Ren J, Wu B, Feng S, Cai G, Tuluc F, Peränen J, Guo W (2015) Activation of Rab8 guanine nucleotide exchange factor Rabin 8 by ERK1/2 in response to EGF signaling. Proc Natl Acad Sci U S A 112:148-153. Medline

Wang T, Liu Y, Xu XH, Deng CY, Wu KY, Zhu J, Fu XQ, He M, Luo ZG (2011) Lgl1 activation of rab10 promotes axonal membrane trafficking underlying neuronal polarization. Dev Cell 21:431-444. CrossRef Medline

Westlake CJ, Baye LM, Nachury MV, Wright KJ, Ervin KE, Phu L, Chalouni C, Beck JS, Kirkpatrick DS, Slusarski DC, Sheffield VC, Scheller RH, Jackson PK (2011) Primary cilia membrane assembly is initiated by Rab11 and transport protein particle II (TRAPPII) complex-dependent trafficking of Rabin8 to the centrosome. Proc Natl Acad Sci U S A 108: 2759-2764. CrossRef Medline

Xie Z, Sanada K, Samuels BA, Shih H, Tsai LH (2003) Serine 732 phosphorylation of FAK by Cdk5 is important for microtubule organization, nuclear movement, and neuronal migration Cell 114:469-482. CrossRef

Xu J, Fotouhi M, McPherson PS (2015) Phosphorylation of the exchange factor DENND3 by ULK in response to starvation activates Rab12 and induces autophagy. EMBO Rep 16:709-718. CrossRef Medline

Yamamura R, Nishimura N, Nakatsuji H, Arase S, Sasaki T (2008) The interaction of JRAB/MICAL-L2 with Rab8 and Rab13 coordinates the assembly of tight junctions and adherens junctions. Mol Biol Cell 19:971983. CrossRef Medline

Yoshimura S, Gerondopoulos A, Linford A, Rigden DJ, Barr FA (2010) Family-wide characterization of the DENN domain Rab GDP-GTP exchange factors. J Cell Biol 191:367-381. CrossRef Medline

Zerial M, McBride H (2001) Rab proteins as membrane organizers. Nat Rev Mol Cell Biol 2:107-117. CrossRef Medline 